\title{
Chiral Effective-Field Theory in the $\Delta(1232)$ Region: Pion Electroproduction on the Nucleon
}

\author{
Vladimir Pascalutsa* and Marc Vanderhaeghen \\ Physics Department, The College of William \&5 Mary, Williamsburg, VA 23187, USA \\ Theory Center, Jefferson Lab, 12000 Jefferson Ave, Newport News, VA 23606, USA
}

(Dated: October 7, 2018)

\begin{abstract}
We develop an extension of chiral perturbation theory to the $\Delta(1232)$-resonance energy region and apply it to investigate the pion electroproduction off the nucleon $\left(e^{-} N \rightarrow e^{-} N \pi\right)$. We present a complete calculation of this process, in the $\Delta$-resonance region, up to next-to-leading order in the $\delta$-expansion. At this order, the only free parameters are the three low-energy constants corresponding to the magnetic (M1), electric (E2), and Coulomb (C2) $\gamma N \rightarrow \Delta$ transition strength. After fitting these parameters to a few well-known data, our calculation provides a prediction for observables and multipole amplitudes of pion electroproduction. These results compare favorably with the phenomenological multipole solutions and recent experimental results from MIT-Bates and MAMI. Our prediction for the pion-mass dependence of the $\gamma N \Delta$ form factors offers an explanation for the discrepancy between the recent lattice-QCD results and the experimental value for the "C2/M1 ratio" at low $Q^{2}$.
\end{abstract}

PACS numbers: 12.39.Fe, 13.40.Gp, 13.60.Le

*Electronic address: vlad@jlab.org

$\dagger$ Electronic address: marcvdh@jlab.org 


\section{INTRODUCTION}

The first excited state of the nucleon - the $\Delta(1232)$ resonance - dominates pionproduction phenomena and plays an important role in our understanding of the low-energy nucleon structure. High-precision measurements of the nucleon-to- $\Delta$ transition by means of electromagnetic probes became possible with the advent of the new generation of electron scattering facilities, such as BATES, MAMI, and JLab, many measurements being completed in recent years [1, 2, 3, 3$]$.

The electromagnetic nucleon-to- $\Delta$ (or, in short $\gamma N \Delta$ ) transition is predominantly of the magnetic dipole $(M 1)$ type. In a simple quark-model picture, this $M 1$ transition is described by a spin flip of a quark in the $s$-wave state. Any $d$-wave admixture in the nucleon or the $\Delta$ wave-functions allows for the electric $(E 2)$ and Coulomb $(C 2)$ quadrupole transitions. Therefore by measuring these one is able to assess the presence of the $d$-wave components and hence quantify to which extent the nucleon or the $\Delta$ wave-function deviates from the spherical shape, i.e., to which extent they are "deformed" [5]. The $d$-wave component of $\Delta$ 's wave-function can be separately assessed by measuring its electric quadrupole moment. However, this would be extremely difficult because of the tiny lifetime of the $\Delta$. The $\gamma N \Delta$ transition, on the other hand, was accurately measured in the pion photo- and electro-production reactions in the $\Delta$-resonance energy region. The $E 2$ and $C 2$ transitions were found to be relatively small at moderate momentum-transfers $\left(Q^{2}\right)$, the ratios $R_{E M}=E 2 / M 1$ and $R_{S M}=C 2 / M 1$ are at the level of a few percent.

Traditionally, the resonance parameters are extracted by using unitary isobar models [6, 1, 8, 9, 10, 11, 12], which in essence are unitarized tree-level calculations based on phenomenological Lagrangians. However, at low $Q^{2}$ the $\gamma N \Delta$-transition shows great sensitivity to the "pion cloud", which until recently could only be comprehensively studied within dynamical models [13, 14, 15, 16, 17, 18]. (Unlike the isobar models, dynamical models include quantum effects due to pion loops.)

With the advent of the chiral effective field theory ( $\chi \mathrm{EFT}$ ) of QCD [19, 20] and its extensions to the $\Delta$-resonance region [21, 22], it has become possible to study the nucleon and $\Delta$-resonance properties in a profoundly different way. Recently, we have been able to perform first $\chi$ EFT studies of the $\gamma N \Delta$-transition in pion electroproduction [23] and of the $\Delta$ resonance magnetic moment in the radiative pion photoproduction [24]. The advantages over the previous dynamical approaches are apparent: $\chi \mathrm{EFT}$ is a low-energy effective field theory of QCD and as such it provides a firm theoretical foundation, with all the relevant symmetries and scales of QCD built in consistently. Moreover, we find that already at next-to-leading order (NLO) in the " $\delta$-expansion" of Ref. 22], the observables for pion electroproduction in the $\Delta$-resonance region are described remarkably well. The $\chi \mathrm{EFT}$, therefore, provides a theoretically consistent and phenomenologically viable framework, which, in particular, will allow for a model-independent extraction of the resonance parameters.

Tremendous progress has recently been achieved as well in the lattice QCD simulations of the $\gamma N \Delta$ transition. The present state-of-the-art results [25] are "quenched" and are obtained for pion masses above $300 \mathrm{MeV}$. These results can only be confronted with experiment after an extrapolation down to the physical pion mass of $140 \mathrm{MeV}$. A linear in the quark mass $\left(m_{q} \sim m_{\pi}^{2}\right)$ extrapolation was used in Ref. [25]. The thus obtained $R_{S M}$ ratio, at low $Q^{2}$, was found to be in major disagreement with experiment. The apparent caveat of this result is that the extrapolation in the quark mass needs not to be linear. The non-analytic dependencies, such as $\sqrt{m_{q}}$ and $\ln m_{q}$, are known to be important as one approaches the 
small physical value of $m_{q}$. These non-analytic terms can also be obtained from $\chi$ EFT, and, as we have demonstrated [23], the $R_{E M}$ and $R_{S M}$ ratios do exhibit a pronounced nonanalytic quark-mass dependence, such that the lattice results [25] can be reconciled with experiment. Here we shall refine our calculation by consistently including the quark-mass dependence of the nucleon and $\Delta$-isobar masses in the same $\chi$ EFT framework [26].

A brief account of this work has recently been published [23]. In this paper we present an extensive description of the work, improve on our theoretical error analysis, and present some new results that could not be included in the brief publication. This paper is organized as follows.

In Sec. III we recall the relevant chiral Lagrangians, while Sec. III explains the power counting in the $\delta$-expansion scheme based on which the leading- and next-to-leading-order contributions are selected. The chiral-loop contributions to the $\gamma N \Delta$-transition form factors are evaluated in Sec. IV by using two different techniques. In Sec. $\nabla$ we discuss the theoretical uncertainty of our calculation due to the neglect of higher-order effects. In Sec. VI we present the results for pion photo- and electroproduction observables, multipoles, and the extracted $\gamma N \Delta$ form factors. In that section we also discuss the $\chi \mathrm{EFT}$ predictions for the $m_{\pi}$-dependence of the $\gamma N \Delta$ transition and compare them with available lattice results. Sec. VII lists the main points and conclusions of the paper. The two Appendices contain technical details concerning the Feynman-parameter and dispersion integrals, respectively.

\section{THE EFFECTIVE CHIRAL LAGRANGIAN}

In this section we define the relevant effective Lagrangian of low-energy QCD, where we restrict ourselves to the two flavor, isospin symmetric case $\left(m_{u}=m_{d} \equiv m_{a}\right)$. The guiding principle is the symmetry under the $\mathrm{SU}(2)_{L} \times \mathrm{SU}(2)_{R}$ chiral rotations, see [19, 20, 27]. The lowest-order Lagrangian of the Goldstone-boson (pion) isovector field $\pi^{a}(a=\overline{1,3})$

$$
\mathcal{L}_{\pi}^{(2)}=\frac{1}{4} f_{\pi}^{2} \operatorname{Tr}\left[\partial_{\mu} U \partial^{\mu} U^{\dagger}+\left(U+U^{\dagger}\right) \chi\right]=\frac{1}{2} \partial_{\mu} \pi^{a} \partial^{\mu} \pi^{a}-\frac{1}{2} m_{\pi}^{2}\left[\pi^{2}+O\left(\pi^{4}\right)\right],
$$

where

$$
U(x)=e^{i \pi^{a}(x) \tau^{a} / f_{\pi}} \equiv u^{2}(x), \quad \text { with } \pi^{a} \tau^{a}=\left(\begin{array}{cc}
\pi^{0} & \sqrt{2} \pi^{+} \\
\sqrt{2} \pi^{-} & -\pi^{0}
\end{array}\right),
$$

and the linear in $U$, explicit symmetry-breaking term is proportional to the quark (or, pion) masses: $\chi=2 B m_{q}=m_{\pi}^{2}+O\left(m_{\pi}^{4}\right)$. Furthermore, at this lowest order, $f_{\pi} \simeq 92.4 \mathrm{MeV}$ is the pion decay constant and $B$ is related to the scalar quark condensate as $B=-\langle\bar{q} q\rangle / f_{\pi}^{2}$. In the notation $\mathcal{L}^{(i)}$, the superscript stands for the number of derivatives of Goldstone-boson fields and insertions of their mass.

The chirally symmetric Lagrangian for the nucleon isodoublet field, $N=(p, n)^{T}$, can conveniently be written by using the $\mathrm{SU}(2)$ vector and axial-vector currents,

$$
\begin{aligned}
& \frac{1}{2} \tau^{a} v_{\mu}^{a}(x) \equiv \frac{1}{2 i}\left(u \partial_{\mu} u^{\dagger}+u^{\dagger} \partial_{\mu} u\right)=\frac{1}{4 f_{\pi}^{2}} \varepsilon^{a b c} \tau^{a} \pi^{b} \partial_{\mu} \pi^{c}+O\left(\pi^{4}\right), \\
& \frac{1}{2} \tau^{a} a_{\mu}^{a}(x) \equiv \frac{1}{2 i}\left(u^{\dagger} \partial_{\mu} u-u \partial_{\mu} u^{\dagger}\right)=\frac{1}{2 f_{\pi}} \tau^{a} \partial_{\mu} \pi^{a}+O\left(\pi^{3}\right)
\end{aligned}
$$

and the chiral covariant derivative acting on the nucleon field,

$$
D_{\mu} N=\partial_{\mu} N+\frac{1}{2} i \tau^{a} v_{\mu}^{a} N
$$


In terms of these definitions the nucleon Lagrangian begins at

$$
\mathcal{L}_{N}^{(1)}=\bar{N}\left(i \not D-M_{N}+\frac{1}{2} g_{A} \tau^{c} \not c^{c} \gamma_{5}\right) N
$$

where $g_{A} \simeq 1.267$ is the nucleon axial-coupling constant.

In the presence of the electromagnetic field $\left(A_{\mu}\right)$, the charge of the pions is accounted for by making the "minimal substitution": $\partial_{\mu} \pi^{a} \rightarrow \partial_{\mu} \pi^{a}+e \varepsilon^{a b 3} A_{\mu} \pi^{b}$, in the above expressions. Similarly, the proton charge is included by the minimal substitution in the chiral derivative as: $D_{\mu} N \rightarrow D_{\mu} N+i e \frac{1}{2}\left(1+\tau^{3}\right) A_{\mu} N$.

The Lagrangian for the spin-3/2 isospin-3/2 $\Delta$-isobar can be written in terms of the Rarita-Schwinger (vector-spinor) isoquartet field ${ }^{1}, \Delta_{\mu}=\left(\Delta_{\mu}^{++}, \Delta_{\mu}^{+}, \Delta_{\mu}^{0}, \Delta_{\mu}^{-}\right)^{T}$, by using the vector and axial-vector currents Eq. (3) in the isospin-3/2 representation of $\mathrm{SU}(2)$. The corresponding generators $\mathcal{T}^{a}$ have the following matrix representation:

$$
\begin{aligned}
\mathcal{T}^{1} & =\frac{2}{3}\left(\begin{array}{cccc}
0 & \sqrt{3} / 2 & 0 & 0 \\
\sqrt{3} / 2 & 0 & 1 & 0 \\
0 & 1 & 0 & \sqrt{3} / 2 \\
0 & 0 & \sqrt{3} / 2 & 0
\end{array}\right), \\
\mathcal{T}^{2} & =\frac{2 i}{3}\left(\begin{array}{cccc}
0 & -\sqrt{3} / 2 & 0 & 0 \\
\sqrt{3} / 2 & 0 & -1 & 0 \\
0 & 1 & 0 & -\sqrt{3} / 2 \\
0 & 0 & \sqrt{3} / 2 & 0
\end{array}\right), \\
\mathcal{T}^{3} & =\operatorname{diag}\left(1, \frac{1}{3},-\frac{1}{3},-1\right),
\end{aligned}
$$

and satisfy $\mathcal{T}^{a} \mathcal{T}^{a}=5 / 3$. The chiral derivative is then given by:

$$
D_{\mu} \Delta_{\nu}=\partial_{\mu} \Delta_{\nu}+i v_{\mu}^{a} \mathcal{T}^{a} \Delta_{\nu}
$$

and the first-order chiral Lagrangian can be written as:

$$
\mathcal{L}_{\Delta}^{(1)}=\bar{\Delta}_{\mu}\left(i \gamma^{\mu \nu \rho} D_{\rho}-M_{\Delta} \gamma^{\mu \nu}\right) \Delta_{\nu}-\frac{1}{2} H_{A} \bar{\Delta}_{\mu} \phi^{c} \mathcal{T}^{c} \gamma_{5} \Delta^{\mu}
$$

where $M_{\Delta} \simeq 1.232 \mathrm{GeV}$ is the mass of the $\Delta$-isobar, $H_{A}$ is the axial coupling constant given, in the large- $N_{C}$ limit [28], by $H_{A}=(9 / 5) g_{A}$ and this value is known to be consistent with the empirical information, see, e.g., [29]. The totally-antisymmetric products of $\gamma$-matrices are defined as: $\gamma^{\mu \nu}=\frac{1}{2}\left[\gamma^{\mu}, \gamma^{\nu}\right], \gamma^{\mu \nu \alpha}=i \varepsilon^{\mu \nu \alpha \beta} \gamma_{\beta} \gamma_{5}$. The electric charge of the $\Delta$ is accounted for by the following minimal substitution: $D_{\mu} \rightarrow D_{\mu}+i e \frac{1}{2}\left(1+3 \mathcal{T}^{3}\right) A_{\mu}$.

The free Rarita-Schwinger field obeys the following field equation:

$$
i \gamma^{\mu \nu \rho} \partial_{\rho} \Delta_{\nu}=M_{\Delta} \gamma^{\mu \nu} \Delta_{\nu}
$$

which, in particular, yields the constraints: $\gamma^{\mu} \Delta_{\mu}=0=\partial^{\mu} \Delta_{\mu}$. The constraints reduce the number of spin degrees of freedom of the vector-spinor field to the physical number appropriate for a massive particle with spin $3 / 2$. These constraints arise essentially due to

\footnotetext{
${ }^{1}$ Frequently chiral Lagrangians for the $\Delta$-isobar are written in terms of the isovector-isodoublet field [21]: $\Delta_{\mu}^{a}=T^{a} \Delta_{\mu}$, with $T$ isospin-1/2-to-3/2 transition matrices. It is an alternative to the isoquartet representation adopted in this work. The two representations, however, are equivalent at the level of observables.
} 
the invariance of the free-field kinetic term [1.h.s. of Eq. (9)], upon the gauge transformation of the spin-3/2 field:

$$
\Delta_{\mu}(x) \rightarrow \Delta_{\mu}(x)+\partial_{\mu} \epsilon(x),
$$

where $\epsilon$ is a spinor. In order for interactions to support the number of the free-field constraints, they must be symmetric with respect to the same (or, at least, similar) transformation [30]. Now, the chiral interactions in Eq. (8) obviously do not have this symmetry. Therefore, we redefine them by using the free field equation, or, equivalently, redefine the spin 3/2 field, such that they become symmetric [31]. Then, e.g., the lowest-order axial coupling becomes

$$
\mathcal{L}_{\Delta \Delta \pi}^{(1)}=-\frac{H_{A}}{2 M_{\Delta} f_{\pi}} \varepsilon^{\mu \nu \rho \sigma} \bar{\Delta}_{\mu} \mathcal{T}^{a}\left(\partial_{\rho} \Delta_{\nu}\right) \partial_{\sigma} \pi^{a}+O\left(\pi^{3}\right) .
$$

The difference with the original interaction term from Eq. (8) is of higher order (in the pion derivatives and mass).

We write the $N \Delta$-transition Lagrangian right away in such an expanded form which exhibits the spin-3/2 gauge symmetry:

$$
\begin{aligned}
\mathcal{L}_{N \Delta}^{(1)} & =\frac{i h_{A}}{2 f_{\pi} M_{\Delta}} \bar{N} T^{a} \gamma^{\mu \nu \lambda}\left(\partial_{\mu} \Delta_{\nu}\right) \partial_{\lambda} \pi^{a}+\text { H.c., } \\
\mathcal{L}_{N \Delta}^{(2)} & =\frac{3 i e g_{M}}{2 M_{N}\left(M_{N}+M_{\Delta}\right)} \bar{N} T^{3} \partial_{\mu} \Delta_{\nu} \tilde{F}^{\mu \nu}+\text { H.c., } \\
\mathcal{L}_{N \Delta}^{(3)} & =\frac{-3 e}{2 M_{N}\left(M_{N}+M_{\Delta}\right)} \bar{N} T^{3} \gamma_{5}\left[g_{E}\left(\partial_{\mu} \Delta_{\nu}\right)+\frac{i g_{C}}{M_{\Delta}} \gamma^{\alpha}\left(\partial_{\alpha} \Delta_{\nu}-\partial_{\nu} \Delta_{\alpha}\right) \partial_{\mu}\right] F^{\mu \nu}+\text { H.c., }(1)
\end{aligned}
$$

where $F^{\mu \nu}$ and $\tilde{F}^{\mu \nu}$ are the electromagnetic field strength and its dual, $T^{a}$ are the isospin1/2-to-3/2 transition $(2 \times 4)$ matrices:

$$
\begin{aligned}
T^{1} & =\frac{1}{\sqrt{6}}\left(\begin{array}{cccc}
-\sqrt{3} & 0 & 1 & 0 \\
0 & -1 & 0 & \sqrt{3}
\end{array}\right), \\
T^{2} & =\frac{-i}{\sqrt{6}}\left(\begin{array}{cccc}
\sqrt{3} & 0 & 1 & 0 \\
0 & 1 & 0 & \sqrt{3}
\end{array}\right), \\
T^{3} & =\sqrt{\frac{2}{3}}\left(\begin{array}{llll}
0 & 1 & 0 & 0 \\
0 & 0 & 1 & 0
\end{array}\right),
\end{aligned}
$$

satisfying $T^{a} T^{b \dagger}=\delta^{a b}-\frac{1}{3} \tau^{a} \tau^{b}$.

Note that the electric and the Coulomb $\gamma N \Delta$ couplings are of one order higher than the magnetic one, because of the $\gamma_{5}$ which involves the "small components" of the fermion fields and thus introduces an extra power of the 3-momentum.

For the momentum-space $\Delta$ propagator we use

$$
S^{\alpha \beta}(p)=\frac{\not p+M_{\Delta}}{M_{\Delta}^{2}-p^{2}}\left[g^{\alpha \beta}-\frac{1}{3} \gamma^{\alpha} \gamma^{\beta}+\frac{(1-\zeta)\left(\zeta \not p+M_{\Delta}\right)}{3\left(\zeta^{2} p^{2}-M_{\Delta}^{2}\right)}\left(\gamma^{\alpha} p^{\beta}-\gamma^{\beta} p^{\alpha}\right)+\frac{2\left(1-\zeta^{2}\right) p^{\alpha} p^{\beta}}{3\left(\zeta^{2} p^{2}-M_{\Delta}^{2}\right)}\right],
$$

where $\zeta$ is a gauge-fixing parameter, see Refs. [26, 32] for details. The analog of the Landaugauge for spin-1 case here is, $\zeta=\infty$ :

$$
S^{\alpha \beta}(p)=\frac{\not p+M_{\Delta}}{M_{\Delta}^{2}-p^{2}} \mathcal{P}^{(3 / 2) \alpha \beta}(p),
$$


with

$$
\mathcal{P}^{(3 / 2) \alpha \beta}(p)=\frac{2}{3}\left(g^{\alpha \beta}-\frac{p^{\alpha} p^{\beta}}{p^{2}}\right)+\frac{\not p}{3 p^{2}} \gamma^{\alpha \beta \mu} p_{\mu},
$$

the covariant spin-3/2 projection operator. As long as the coupling of the spin-3/2 field are gauge-symmetric with respect to the transformation (10), the results are independent of the choice of the gauge-fixing parameter $\zeta$.

\section{POWER COUNTING AND RENORMALIZATION}

The inclusion of the $\Delta$-resonance introduces another light scale in the theory, the resonance excitation energy: $\Delta \equiv M_{\Delta}-M_{N} \sim 0.3 \mathrm{GeV}$. This energy scale is still relatively light in comparison to the chiral symmetry breaking scale $\Lambda_{\chi S B} \sim 1 \mathrm{GeV}$. Therefore, $\delta=\Delta / \Lambda_{\chi S B}$ can be treated as a small parameter. The question is, how to compare this parameter with the small parameter of chiral perturbation theory $(\chi \mathrm{PT}), \epsilon=m_{\pi} / \Lambda_{\chi S B}$. After all, our aim is to organize an expansion in a small parameter and to estimate the size of various contributions based on power-counting rules. So some specific relation between $\delta$ and $\epsilon$ would be helpful.

In most of the literature (see, e.g., [21]) they are assumed to be of comparable size, $\delta \approx \epsilon$. This, however, leads to a somewhat unsatisfactory result: the $\Delta$-resonance contributions are always estimated to be of the same size as the nucleon contributions. Thus, obviously, the $\Delta$-contributions are overestimated at lower energies and underestimated at the resonance energies. To estimate the $\Delta$-resonance contributions correctly, and depending on the energy region, one needs to count $\delta$ and $\epsilon$ differently.

A relation $\epsilon=\delta^{2}$ was suggested and explored in Ref. [22], and is adopted in this work. The second power is indeed the closest integer power for the relation of these parameters in the real world. We should stress that this relation is used for power-counting purposes only. It is not imposed in the actual evaluations of diagrams. Each diagram is simply characterized by an overall $\delta$-counting index $n$, which tells us that its contribution begins at $\mathrm{O}\left(\delta^{n}\right)$.

Because of the distinction of $m_{\pi}$ and $\Delta$ the counting of a given diagram depends on whether the characteristic momentum $p$ is in the low-energy region $\left(p \sim m_{\pi}\right)$ or in the resonance region $(p \sim \Delta)$. In the low-energy region the index of a graph with $L$ loops, $N_{\pi}$ pion propagators, $N_{N}$ nucleon propagators, $N_{\Delta} \Delta$-propagators, and $V_{i}$ vertices of dimension $i$ is

$$
n=2\left(\sum_{i} i V_{i}+4 L-N_{N}-2 N_{\pi}\right)-N_{\Delta} \equiv 2 n_{\chi \mathrm{P} T}-N_{\Delta},
$$

where $n_{\chi \mathrm{P} T}$ is the index in $\chi \mathrm{PT}$ with no $\Delta^{\prime} \mathrm{s}$ [27]. In the resonance region, one distinguishes the one- $\Delta$-reducible $(\mathrm{O} \Delta \mathrm{R})$ graphs 22 ], see e.g., graph (a) in Fig. 2] Such graphs contain $\Delta$ propagators which go as $1 /(p-\Delta)$, and hence for $p \sim \Delta$ they are large and all need to be included. This gives an incentive, within the power-counting scheme, to resum $\Delta$ contributions. Their resummation amounts to dressing the $\Delta$ propagators so that they behave as $1 /(p-\Delta-\Sigma)$. The self-energy $\Sigma$ begins at order $p^{3}$ and thus a dressed $\mathrm{O} \Delta \mathrm{R}$ propagator counts as $1 / \delta^{3}$. If the number of such propagators in a graph is $N_{O \Delta R}$, the power-counting index of this graph in the resonance region is given by

$$
n=n_{\chi \mathrm{PT}}-N_{\Delta}-2 N_{O \Delta R},
$$

where $N_{\Delta}$ is the total number of $\Delta$-propagators. 
A word on the renormalization program, as it is an indivisible part of power counting in a relativistic theory. Indeed, without some kind of renormalization the loop graphs diverge as $\Lambda^{\mathcal{N}}$, where $\Lambda$ is an ultraviolet cutoff, and $\mathcal{N}$ is a positive power proportional to the powercounting index of the graph. Also, contributions of heavy scales, such as baryon masses, may appear as $M^{\mathcal{N}}$. The renormalization of the loop graphs can and should be performed so as to absorb these large contributions into the available low-energy constants, thus bringing the result in accordance with power counting [33].

To give an example, consider the one- $\pi N$-loop contribution to the nucleon mass, Fig. 1. For the $\pi N N$ vertex from $\mathcal{L}_{N}^{(1)}$ the power counting tells us that this contribution begins at $O\left(m_{\pi}^{3}\right)$. An explicit calculation, however, will show (e.g., 27]) that the loop produces $O\left(m_{\pi}^{0}\right)$ and $O\left(m_{\pi}^{2}\right)$ terms, both of which are (infinitely) large. This is not a violation of power counting, because there are two low-energy constants: the nucleon mass in the chiral limit, $M^{(0)}$, and $c_{1 N}$, which enter at order $O\left(m_{\pi}^{0}\right)$ and $O\left(m_{\pi}^{2}\right)$, respectively, and renormalize away the large contributions coming from the loop. The renormalized relativistic result, up to and including $O\left(m_{\pi}^{3}\right)$, can be written as [26]:

$$
\begin{aligned}
& M_{N}= M_{N}^{(0)}-4 c_{1 N} m_{\pi}^{2} \\
&-\frac{3 g_{A}^{2}}{\left(8 \pi f_{\pi}\right)^{2}} m_{\pi}^{3}\left\{4\left(1-\frac{m_{\pi}^{2}}{4 M_{N}^{2}}\right)^{5 / 2} \arccos \frac{m_{\pi}}{2 M_{N}}+\frac{17 m_{\pi}}{16 M_{N}}-\left(\frac{m_{\pi}}{2 M_{N}}\right)^{3}\right. \\
&\left.+\frac{m_{\pi}}{8 M_{N}}\left[30-10\left(\frac{m_{\pi}}{M_{N}}\right)^{2}+\left(\frac{m_{\pi}}{M_{N}}\right)^{4}\right] \ln \frac{m_{\pi}}{M_{N}}\right\}
\end{aligned}
$$

and one can easily verify that the loop contribution begins at $O\left(m_{\pi}^{3}\right)$ in agreement with power counting.

We now turn to the analysis of the pion electroproduction process. The pion electroproduction amplitude to NLO in the $\delta$ expansion, in the resonance region, is given by graphs in Fig. 2(a) and (b), where the shaded blobs in graph (a) include corrections depicted in Fig. 2( $(\mathrm{c}-\mathrm{f})$. The hadronic part of graph (a) begins at $\mathcal{O}\left(\delta^{0}\right)$ which here is the leading order. The Born graphs Fig. 2(b) contribute at $\mathcal{O}(\delta)$. We note that at NLO there are also vertex corrections of the type (e) and (f) with nucleon propagators in the loop replaced by the $\Delta$ propagators. However, after the appropriate renormalizations and $Q^{2} \ll \Lambda \Delta$, these graphs start to contribute at next-next-to-leading order.

The $\Delta$ self-energy Fig. 2(c) can, in the $\zeta=\infty$ gauge, be presented as

$$
\Sigma_{\alpha \beta}(p)=\Sigma_{\Delta}(\not p) \mathcal{P}_{\alpha \beta}^{(3 / 2)}(p),
$$

where $\Sigma_{\Delta}(\not p)$ has the spin-1/2 Lorentz form, computed by us earlier [24, 26]. To recall these results, it is convenient to introduce the following dimensionless quantities:

$$
\begin{aligned}
& \mu=\frac{m_{\pi}}{M_{\Delta}}, \quad r=\frac{M_{N}}{M_{\Delta}}, \quad \delta=\frac{\Delta}{M_{\Delta}}=1-r, \\
& \beta=\frac{1}{2}\left(1-r^{2}+\mu^{2}\right), \quad \alpha=1-\beta, \\
& \lambda^{2}=\frac{1}{4}\left(\delta^{2}-\mu^{2}\right)\left[(1+r)^{2}-\mu^{2}\right]=\beta^{2}-\mu^{2}=\alpha^{2}-r^{2} .
\end{aligned}
$$

The imaginary part of the $\Delta$ self-energy is related to the resonance decay width, which at the leading order (LO) gives:

$$
\Gamma_{\Delta}=-2 \operatorname{Im} \Sigma_{\Delta}\left(M_{\Delta}\right)=(4 \pi / 3) M_{\Delta} C_{\Delta}^{2} \lambda^{3}(\alpha+r) .
$$


with $C_{\Delta}=h_{A} M_{\Delta} /\left(8 \pi f_{\pi}\right)$. The experimental value for the $\Delta$-resonance width, $\Gamma_{\Delta}=$ $0.115 \mathrm{GeV}$, fixes $h_{A} \simeq 2.85$. At NLO, the residue of the $\Delta$ propagator receives a correction:

$$
\operatorname{Im} \Sigma_{\Delta}^{\prime}\left(M_{\Delta}\right)=-2 \pi C_{\Delta}^{2} \lambda\left[\alpha \beta(\alpha+r)-\frac{1}{3} \lambda^{2}\left(r+r^{2}-\mu^{2}\right)\right] .
$$

The renormalized $\Delta$-propagator, in the $\zeta=\infty$ gauge, at NLO reads:

$$
S_{\alpha \beta}(p)=\frac{-\mathcal{P}_{\alpha \beta}^{(3 / 2)}(p)}{\left(\not p-M_{\Delta}\right)\left[1-i \operatorname{Im} \Sigma_{\Delta}^{\prime}\left(M_{\Delta}\right)\right]+\frac{1}{2} i \Gamma_{\Delta}} .
$$

The real part of the renormalized $\Delta$ self-energy, $\tilde{\Sigma}_{\Delta}$, contributes to the mass as:

$$
M_{\Delta}=M_{\Delta}^{(0)}-4 c_{1 \Delta} m_{\pi}^{2}+\operatorname{Re} \tilde{\Sigma}_{\Delta}^{(\pi N)}\left(M_{\Delta}\right),
$$

where

$$
\tilde{\Sigma}_{\Delta}\left(M_{\Delta}\right)=-\frac{1}{2} C_{\Delta}^{2} M_{\Delta}\left[V(\mu, \delta)-V(0, \delta)-\mu^{2} V^{\prime}(0, \delta)\right] .
$$

The $\pi N$ loop integral $V(\mu, \delta)$ is given in analytical form by [26]:

$$
\begin{aligned}
V(\mu, \delta) & =\frac{1}{3}(r+\alpha)\left[\beta\left(\mu^{2}-2 \lambda^{2}\right) \ln \mu^{2}+\alpha\left(r^{2}-2 \lambda^{2}\right) \ln r^{2}-\frac{2}{3}\left(\alpha^{3}+\beta^{3}\right)\right. \\
& \left.+4 \lambda^{2}+4 \lambda^{4} \Omega(\lambda)\right]+\frac{1}{4} \mu^{4}\left(\ln \mu^{2}-\frac{1}{2}\right)-\frac{1}{4} r^{4}\left(\ln r^{2}-\frac{1}{2}\right),
\end{aligned}
$$

with the elementary function $\Omega$ defined as:

$$
\Omega(\lambda)= \begin{cases}\frac{1}{2 \lambda} \ln \frac{\beta-\mu^{2}-\lambda}{\beta-\mu^{2}+\lambda}, & \lambda^{2} \geq 0 \\ -\frac{1}{\sqrt{-\lambda^{2}}} \arctan \frac{\sqrt{-\lambda^{2}}}{\alpha \beta+\lambda^{2}}, & \lambda^{2}<0\end{cases}
$$

The region $\lambda^{2}>0$, corresponds with $m_{\pi}<M_{\Delta}-M_{N}$, where the $\Delta$ is unstable. The region $\lambda^{2}<0$, corresponds with $m_{\pi}>M_{\Delta}-M_{N}$, where the $\Delta$ is stable.

Furthermore, from Eq. (27) we read off the $m_{\pi}^{0}$ and $m_{\pi}^{2}$ terms, which enter Eq. (26) and need to be absorbed by the renormalization of the low-energy constants,

$$
\begin{aligned}
V(0, \delta) & =\frac{1}{4}\left[\frac{r}{3}\left(2-r^{2}\right)+2\left(1-\frac{r^{2}}{3}\right)\right] r^{5} \ln r^{2}-\frac{1}{12}(1+r)^{2}\left(1-r^{2}\right)^{3} \ln \left(1-r^{2}\right) \\
& +\left[\frac{5}{36}-\frac{7 r^{2}}{36}-\frac{r^{4}}{8}+\frac{r^{6}}{12}+\frac{r}{18}\left(5-12 r^{2}+3 r^{4}\right)\right], \\
V^{\prime}(0, \delta) & =\frac{1}{2}\left(1+\frac{2 r}{3}\right) r^{5} \ln r^{2}+\left[\frac{1}{3}\left(1+r^{2}+r^{4}\right)+\frac{r}{2}\left(1+r^{2}\right)\right]\left(1-r^{2}\right) \ln \left(1-r^{2}\right) \\
& -\left[\frac{7}{18}+\frac{r}{2}+\frac{r^{2}}{6}+\frac{r^{3}}{2}+\frac{r^{4}}{3}\right] .
\end{aligned}
$$

The vector-meson diagram, Fig. 2(d), contributes to NLO for $Q^{2} \sim \Lambda \Delta$. We include it effectively by giving the $g_{M}$-term a dipole $Q^{2}$-dependence (in analogy to how it is usually done for the nucleon isovector form factor):

$$
g_{M} \rightarrow \frac{g_{M}}{\left(1+Q^{2} / 0.71 \mathrm{GeV}^{2}\right)^{2}} .
$$

Analogous effect for the $g_{E}$ and $g_{C}$ couplings begins at $\mathrm{N}^{2} \mathrm{LO}$. The loop corrections to the $\gamma N \Delta$-vertex are discussed separately, in the following section. 


\section{EVALUATION OF THE $\gamma N \Delta$ FORM FACTORS}

In this section we present a detailed analysis of the $\gamma N \Delta$-vertex corrections that appear at NLO, see Fig. 2(e) and (f). To the order we work, the pseudovector $\pi N N$ interaction from the Lagrangian (5) is equivalent to the pseudoscalar one, and we use the latter in the actual calculations of the graphs Fig. 2(e, f).

The rest of the section is organized as follows. First we will comment on the electromagnetic gauge invariance of these contributions. Then, after a brief summary of different decompositions of the $\gamma N \Delta$ vertex into Lorentz-invariant form factors, we evaluate the chiral corrections to those form factors at NLO. As a cross-check, we do the calculations using two different techniques: the Feynman-parameter method and the sideways dispersion relations.

\section{A. Electromagnetic current conservation}

The addition of the one-loop graphs Fig. 2(e, f) to the "tree-level" $\gamma N \Delta$ vertex from Eq. (12), should not spoil the e.m. current conservation:

$$
q_{\mu} \bar{u}_{\alpha}\left(p^{\prime}\right) \Gamma_{\gamma N \Delta}^{\alpha \mu} u(p)=0
$$

where $u_{\alpha}$ is the free Rarita-Schwinger vector-spinor of the $\Delta, u$ is the free Dirac spinor of the nucleon, and momenta are defined as in Fig. 3. This sort of conditions are most conveniently checked by using the Ward-Takahashi (WT) identities for the e.m. couplings of the nucleon, pion and $\Delta$ fields:

$$
\begin{aligned}
& q_{\mu} \Gamma_{\gamma N N}^{\mu}=e_{N} q \cdot \gamma=e_{N}\left[S_{N}^{-1}\left(p^{\prime}\right)-S_{N}^{-1}(p)\right], \\
& q_{\mu} \Gamma_{\gamma \pi \pi}^{\mu}=e_{\pi} q \cdot\left(p+p^{\prime}\right)=e_{\pi}\left[S_{\pi}^{-1}\left(p^{\prime}\right)-S_{\pi}^{-1}(p)\right], \\
& q_{\mu} \Gamma_{\gamma \Delta \Delta}^{\mu \alpha \beta}=e_{\Delta} \gamma^{\mu \alpha \beta} q_{\mu}=e_{\Delta}\left[S_{\Delta}^{-1 \alpha \beta}\left(p^{\prime}\right)-S_{\Delta}^{-1 \alpha \beta}(p)\right],
\end{aligned}
$$

where $q=p^{\prime}-p, e_{N}=\frac{1}{2} e\left(1+\tau_{3}\right), e_{\pi}=i e \epsilon^{a b c} \tau_{c}, e_{\Delta}=\frac{1}{2} e\left(1+3 \mathcal{T}^{3}\right)$ and $S^{-1}$ denote the corresponding inverse propagators. Applying these identities to the diagrams Fig. [2(e, f) we obtain:

$$
q_{\mu} \bar{u}_{\alpha}\left(p^{\prime}\right) \Gamma_{\gamma N \Delta}^{\alpha \mu} u(p)=e \bar{u}_{\alpha}\left(p^{\prime}\right)\left[\Sigma_{N \Delta}^{\alpha}(p)-\Sigma_{N \Delta}^{\alpha}\left(p^{\prime}\right)\right] u(p),
$$

where $\Sigma_{N \Delta}^{\alpha}$ is the one-loop $N \rightarrow \Delta$ self-energy, which, due to Lorentz covariance has the following general form:

$$
\Sigma_{N \Delta}^{\alpha}(p)=A\left(p^{2}\right) p^{\alpha}+B\left(p^{2}\right) \gamma^{\alpha} .
$$

The scalar functions $A$ and $B$ do not vanish, however we may use the Rarita-Schwinger conditions, $p \cdot u(p)=0=\gamma \cdot u(p)$, to show that the second term on the r.h.s. of Eq. (34) vanishes. The first term can only be canceled by the contribution of the diagram in Fig. 4,

Thus, the condition of the e.m. current conservation requires the diagram of Fig. 4 to be included in addition to the NLO contributions Fig. 2(e, f). When computing this diagram, we discover that it is actually of $\mathrm{N}^{2} \mathrm{LO}$, because the Lorentz form Eq. (35) cuts out the spin$3 / 2$ part of the $\Delta$-propagator and hence also the pole of the propagator. The $\Delta$-propagator in Fig. 4, therefore, counts as $\delta^{0}$.

To NLO, the sum of Fig. 2(e) and Fig. 2(f) conserves the e.m. current. 


\section{B. Form-factor decompositions}

The $\gamma N \Delta$ vertex can in general be decomposed into three Lorentz covariants. For instance,

$$
\begin{aligned}
& \bar{u}_{\alpha}\left(p^{\prime}\right) \Gamma_{\gamma N \Delta}^{\alpha \mu} u(p)=\sqrt{\frac{3}{2}} \frac{M_{\Delta}+M_{N}}{M_{N}\left[\left(M_{\Delta}+M_{N}\right)^{2}+Q^{2}\right]} \\
& \times \bar{u}_{\alpha}\left(p^{\prime}\right)\left\{g_{M}\left(Q^{2}\right) \varepsilon^{\alpha \mu \kappa \lambda} p_{\kappa}^{\prime} q_{\lambda}\right. \\
& +g_{E}\left(Q^{2}\right)\left(q^{\alpha} p^{\prime \mu}-q \cdot p^{\prime} g^{\alpha \mu}\right) i \gamma_{5} \\
& \left.+g_{C}\left(Q^{2}\right)\left(q^{\alpha} q^{\mu}-q^{2} g^{\alpha \mu}\right) i \gamma_{5}\right\} u(p),
\end{aligned}
$$

where $q=p^{\prime}-p$ is the photon 4-momentum, $Q^{2}=-q^{2}$. Furthermore, $g_{M}\left(Q^{2}\right), g_{E}\left(Q^{2}\right)$, and $g_{C}\left(Q^{2}\right)$ are the magnetic dipole, electric quadrupole, and Coulomb quadrupole form factors, as defined in Ref. [18]. In the limit $Q^{2}=0$ they are equal to the physical values of the corresponding parameters in the Lagrangian (12). These form factors relate to the conventional magnetic $\left(G_{M}^{*}\right)$, electric $\left(G_{E}^{*}\right)$ and Coulomb $\left(G_{C}^{*}\right)$ form factors of Jones and Scadron [34] as follows:

$$
\begin{aligned}
G_{M}^{*} & =g_{M}+\frac{M_{\Delta}^{2}}{Q_{+}^{2}}\left(-\beta_{\gamma} g_{E}+\bar{Q}^{2} g_{C}\right), \\
G_{E}^{*} & =\frac{M_{\Delta}^{2}}{Q_{+}^{2}}\left(-\beta_{\gamma} g_{E}+\bar{Q}^{2} g_{C}\right), \\
G_{C}^{*} & =-\frac{2 M_{\Delta}^{2}}{Q_{+}^{2}}\left(g_{E}+\beta_{\gamma} g_{C}\right),
\end{aligned}
$$

where $Q_{ \pm}=\sqrt{\left(M_{\Delta} \pm M_{N}\right)^{2}+Q^{2}}, \bar{Q}^{2}=Q^{2} / M_{\Delta}^{2}, \beta_{\gamma}=\frac{1}{2}\left(1-r^{2}-\bar{Q}^{2}\right)$. The multipole ratios $E 2 / M 1$ and $C 2 / M 1$ at the $\Delta$-resonance position can be expressed in terms of these form factors as:

$$
R_{E M}=-\frac{G_{E}^{*}}{G_{M}^{*}}, \quad R_{S M}=-\frac{Q_{+} Q_{-}}{4 M_{\Delta}^{2}} \frac{G_{C}^{*}}{G_{M}^{*}} .
$$

Alternatively, the on-shell $\gamma N \Delta$ vertex is also often expressed in the following form :

$$
\begin{aligned}
\bar{u}_{\alpha}\left(p^{\prime}\right) \Gamma_{\gamma N \Delta}^{\alpha \mu} u(p)=\sqrt{\frac{2}{3}} \bar{u}_{\alpha}\left(p^{\prime}\right)\{ & \left(\gamma^{\mu} q^{\alpha}-\gamma \cdot q g^{\alpha \mu}\right) g_{1}\left(Q^{2}\right) \\
& +\left(q \cdot p^{\prime} g^{\alpha \mu}-q^{\alpha} p^{\prime \mu}\right) g_{2}\left(Q^{2}\right) \\
& \left.+\left(q^{\alpha} q^{\mu}-q^{2} g^{\alpha \mu}\right) g_{3}\left(Q^{2}\right)\right\} i \gamma_{5} u(p),
\end{aligned}
$$

which is defined for the $p \rightarrow \Delta^{+}$transition, yielding the isospin factor $\sqrt{2 / 3}$. The form factors $g_{M}, g_{E}$ and $g_{C}$ can then be expressed in terms of $g_{i}\left(Q^{2}\right)(i=1,2,3)$ through the relations :

$$
\begin{aligned}
g_{M}\left(Q^{2}\right) & =-\frac{2}{3} \frac{M_{N} Q_{+}^{2}}{M_{\Delta}\left(M_{\Delta}+M_{N}\right)} g_{1}\left(Q^{2}\right), \\
g_{E}\left(Q^{2}\right) & =\frac{2}{3} \frac{M_{N} Q_{+}^{2}}{M_{\Delta}\left(M_{\Delta}+M_{N}\right)}\left\{g_{1}\left(Q^{2}\right)-M_{\Delta} g_{2}\left(Q^{2}\right)\right\}, \\
g_{C}\left(Q^{2}\right) & =\frac{2}{3} \frac{M_{N} Q_{+}^{2}}{\left(M_{\Delta}+M_{N}\right)} g_{3}\left(Q^{2}\right) .
\end{aligned}
$$




\section{Feynman-parameter method}

The one-loop corrections to the $\gamma N \Delta$ form factors are given by the graphs in Fig. 2(e) and (f). As they contain three propagators, we apply the Feynman-parameter trick :

$$
\frac{1}{A B C}=2 \int_{0}^{1} d x \int_{0}^{x} d y \frac{1}{[A y+B(x-y)+C(1-x)]^{3}} .
$$

For the diagram Fig. 2(e) we have $A=k^{2}-m_{\pi}^{2}+i \epsilon, B=(k+q)^{2}-m_{\pi}^{2}+i \epsilon, C=$ $(p-k)^{2}-M_{N}^{2}+i \epsilon$, where $k$ is the integration 4-momentum. After the shift of the integration momentum, $k \rightarrow k-(x-y) q+(1-x) p$, we obtain : $A y+B(x-y)+C(1-x) \rightarrow k^{2}-\mathcal{M}^{2}$, where

$$
\mathcal{M}^{2}(x, y)=m_{\pi}^{2} x+\left(M_{N}^{2}-p^{2} x\right)(1-x)-2 p \cdot q(1-x)(x-y)-q^{2}(x-y)(1-x-y)-i \epsilon .
$$

For the case of the $N \rightarrow \Delta$ transition we may use: $p^{2}=M_{N}^{2}, p^{\prime 2}=(p+q)^{2}=M_{\Delta}^{2}$, and hence $2 p \cdot q=M_{\Delta}^{2}-M_{N}^{2}-q^{2}$, and

$$
\mathcal{M}^{2}(x, y)=m_{\pi}^{2} x+\left[M_{N}^{2}-x M_{\Delta}^{2}+\left(M_{\Delta}^{2}-M_{N}^{2}\right) y\right](1-x)+q^{2} y(x-y)-i \epsilon .
$$

The next step is to perform the integration over 4-momentum which can be done by using the following rules:

$$
\begin{aligned}
& \int \frac{d^{4} k}{(2 \pi)^{4}} \frac{1}{\left(k^{2}-m^{2}\right)^{n}} \equiv J_{n}\left(m^{2}\right)=i \frac{(-1)^{n}}{(4 \pi)^{2}} \frac{\Gamma(n-2)}{\Gamma(n)} m^{-2(n-2)}, \\
& \int \frac{d^{4} k}{(2 \pi)^{4}} \frac{k_{\mu} k_{\nu}}{\left(k^{2}-m^{2}\right)^{n}}=\frac{1}{2(n-1)} J_{n-1}\left(m^{2}\right) g_{\mu \nu},
\end{aligned}
$$

Integrals with an odd number of 4 -vectors $k$ in the numerator vanish. The integral $J_{n}$ diverges for $n=1$ and 2, but can be defined via dimensional regularization as:

$$
\begin{aligned}
& J_{1}\left(m^{2}\right)=\frac{-i m^{2}}{(4 \pi)^{2}}\left(-\frac{2}{4-d}+\gamma_{E}-1+\ln \frac{m^{2}}{4 \pi}\right), \\
& J_{2}\left(m^{2}\right)=\frac{-i}{(4 \pi)^{2}}\left(-\frac{2}{4-d}+\gamma_{E}+\ln \frac{m^{2}}{4 \pi}\right),
\end{aligned}
$$

where $d \rightarrow 4^{-}$is the number of dimensions and $\gamma_{E}=-\Gamma^{\prime}(1) \simeq 0.5772$ is the Euler constant.

Then, the $(\overline{M S}$-subtracted) result for the graph of Fig. 2(e), where the photon couples to the charged pion in the loop, can be decomposed into the three Lorentz covariants Eq. (36) and cast in the form :

$$
\begin{aligned}
g_{M}^{(e)} & =-C_{N \Delta} \int_{0}^{1} d y \int_{0}^{1-y} d x \ln \mathcal{M}_{e}^{2} \\
g_{E}^{(e)} & =+C_{N \Delta} \int_{0}^{1} d y y \int_{0}^{1-y} d x\left\{\ln \mathcal{M}_{e}^{2}-2 x[(1-y)(1+r)-x] \mathcal{M}_{e}^{-2}\right\}, \\
g_{C}^{(e)} & =-C_{N \Delta} \int_{0}^{1} d y y(2 y-1) \int_{0}^{1-y} d x[(1-y)(1+r)-x] \mathcal{M}_{e}^{-2}, \\
\text { with } & \mathcal{M}_{e}^{2} \equiv(x-\beta)^{2}-\lambda^{2}+2 \beta_{\gamma} x y+\bar{Q}^{2} y(1-y)-i \varepsilon,
\end{aligned}
$$


while the analogous contribution of the graph Fig. 2(f), where the photon couples to the electric charge of the nucleon in the loop, is given by :

$$
\begin{aligned}
& g_{M}^{(f)}=-C_{N \Delta} \int_{0}^{1} d y y \int_{0}^{1-y} d x\left\{2 \ln \mathcal{M}_{f}^{2}\right. \\
&\left.+\mathcal{M}_{f}^{-2}\left[2 \alpha_{\gamma} x y+\bar{Q}^{2} y(1-y)-(x+r)(1-x)\right]\right\}, \\
& g_{E}^{(f)}=-g_{M}^{(f)}+2 C_{N \Delta} \int_{0}^{1} d y y \int_{0}^{1-y} d x \mathcal{M}_{f}^{-2}[(x+r)(1-x)-x y(1+r)], \\
& g_{C}^{(f)}=-2 C_{N \Delta} \int_{0}^{1} d y y^{2} \int_{0}^{1-y} d x[y(1+r)-1+x] \mathcal{M}_{f}^{-2}, \\
& \text { with } \quad \mathcal{M}_{f}^{2} \equiv(x-\alpha)^{2}-\lambda^{2}+2 \alpha_{\gamma} x y+\bar{Q}^{2} y(1-y)-i \varepsilon,
\end{aligned}
$$

where we have used the definitions of Eq. (21) and introduced,

$$
C_{N \Delta}=\frac{4 g_{A} h_{A}}{\left(8 \pi f_{\pi}\right)^{2}} \frac{Q_{+}^{2} r^{2}}{3(1+r)}
$$

Finally, the integration over $x$ and some of the $y$-integration can be done analytically, see Appendix A.

\section{Dispersion method}

Alternatively, we can compute the correction to the $\gamma N \Delta$ vertex by exploiting the analyticity of the loop contributions. We use the so-called sideways dispersion relations [35, 36], and hence start with calculating the absorptive (imaginary) part of the $\gamma N \Delta$ vertex from the cut in the $\pi N$-loop diagrams of Fig. 5. Subsequently, the real part of the on-shell $\gamma N \Delta$ vertex is computed through the dispersion relation in the $\Delta$-resonance 4-momentum squared $p^{2}$.

We first express the half off-shell $\gamma N \Delta$ vertex (where we allow the $\Delta$ to be off-shell) in terms of the invariants $g_{i}\left(s={p^{\prime}}^{2}, Q^{2}\right)$, for $i=1,2,3$, as :

$$
\begin{aligned}
\Gamma^{\alpha \mu} u(p)=\left(\mathcal{P}_{+}^{3 / 2}\left(p^{\prime}\right)\right)_{\beta}^{\alpha} & \left\{\left(\gamma^{\mu} q^{\beta}-\gamma \cdot q g^{\beta \mu}\right) g_{1}^{+}\left(s, Q^{2}\right)\right. \\
& +\left(q \cdot p^{\prime} g^{\beta \mu}-q^{\beta} p^{\prime \mu}\right) g_{2}^{+}\left(s, Q^{2}\right) \\
& \left.+\left(q^{\beta} q^{\mu}-q^{2} g^{\beta \mu}\right) g_{3}^{+}\left(s, Q^{2}\right)\right\} i \gamma_{5} u(p) \\
+\left(\mathcal{P}_{-}^{3 / 2}\left(p^{\prime}\right)\right)_{\beta}^{\alpha}\{ & \left(\gamma^{\mu} q^{\beta}-\gamma \cdot q g^{\beta \mu}\right) g_{1}^{-}\left(s, Q^{2}\right) \\
+ & \left(q \cdot p^{\prime} g^{\beta \mu}-q^{\beta} p^{\prime \mu}\right) g_{2}^{-}\left(s, Q^{2}\right) \\
& \left.+\left(q^{\beta} q^{\mu}-q^{2} g^{\beta \mu}\right) g_{3}^{-}\left(s, Q^{2}\right)\right\} i \gamma_{5} u(p)
\end{aligned}
$$

where the spin-3/2 positive (negative) energy projectors are given by

$$
\left(\mathcal{P}_{ \pm}^{3 / 2}\left(p^{\prime}\right)\right)_{\alpha \beta}=\frac{\left(\gamma \cdot p^{\prime} \pm M_{\Delta}\right)}{2 M_{\Delta}}\left\{g_{\alpha \beta}-\frac{1}{3} \gamma_{\alpha} \gamma_{\beta}-\frac{1}{3 p^{\prime 2}}\left(\gamma \cdot p^{\prime} \gamma_{\alpha} p_{\beta}^{\prime}+p_{\alpha}^{\prime} \gamma_{\beta} \gamma \cdot p^{\prime}\right)\right\}
$$


We next determine the real parts of the invariants $g_{i}^{+}$for $s=M_{\Delta}^{2}$ (i.e., the $\Delta$ is on-shell) through a dispersion relation in $s$ as :

$$
\operatorname{Re} g_{i}^{+}\left(M_{\Delta}^{2}, Q^{2}\right)=\frac{1}{\pi} \mathrm{P} \int_{s_{\text {th }}}^{\infty} d s \frac{\operatorname{Im} g_{i}^{+}\left(s, Q^{2}\right)}{s-M_{\Delta}^{2}},
$$

where the integration starts from the $\pi N$ threshold $s_{t h}=\left(M_{N}+m_{\pi}\right)^{2}$, and where $\mathrm{P}$ denotes the principal value integration. These unsubstracted dispersion integrals do not converge. However, the dispersion relations for the following differences

$$
\operatorname{Re} g_{i}^{+}\left(M_{\Delta}^{2}, Q^{2}\right)-\operatorname{Re} g_{i}^{+}\left(M_{\Delta}^{2}, 0\right)=\frac{1}{\pi} \mathrm{P} \int_{s_{t h}}^{\infty} d s \frac{\operatorname{Im} g_{i}^{+}\left(s, Q^{2}\right)-\operatorname{Im} g_{i}^{+}(s, 0)}{s-M_{\Delta}^{2}}
$$

do converge, for all the considered contributions. This subtraction can be put in correspondence with the renormalization of appropriate low-energy constants from the Lagrangian (12).

The expressions for the absorptive (imaginary) parts of these form factors are calculated in Appendix $\mathrm{B}$ with the results given by Eqs. (B50), (B51). The dispersion integrals in Eq. (52) are evaluated numerically. The physical form factors are then simply given by $g_{i}\left(Q^{2}\right)=g_{i}^{+}\left(M_{\Delta}^{2}, Q^{2}\right)$.

We compared the thus obtained results with the Feynman-parameter method results. We found perfect agreement between both methods for all three $\gamma N \Delta$ transition form factors, which provides a cross-check on our calculations.

\section{ERRORS DUE TO NEGLECT OF N2LO EFFECTS}

Prior to presenting the results of our NLO calculation, we would like to make an estimate of the theoretical uncertainty due to the neglect of higher-order effects. Some of the nextnext-to-leading order $\left(\mathrm{N}^{2} \mathrm{LO}\right)$ contributions are shown in Fig. 6. Of course, there is no substitute for an actual calculation of those effects, but at present we only know that they must be suppressed by at least one power of $\delta\left(=\Delta / \Lambda_{\chi S B}\right)$ as compared to the NLO and two powers of $\delta$ as compared to the LO contributions. Therefore, we can estimate the size of the $\mathrm{N}^{2} \mathrm{LO}$ contribution to an amplitude $A$ as: $A_{\mathrm{NLO}} \delta$, or $A_{\mathrm{LO}} \delta^{2}$. The theoretical uncertainty of a calculation up to and including NLO can thus be estimated as:

$$
A_{\text {err }}=\left|A_{\mathrm{LO}}+A_{\mathrm{NLO}}\right| \delta^{2} .
$$

In cases where the amplitude does not receive any LO contributions, we have no other option than,

$$
A_{\text {err }}=\left|A_{\mathrm{NLO}}\right| \delta
$$

This looks nice and simple, however, there are a few caveats in the implementation of such an estimate. First of all, although we have introduced $\delta$ as $\Delta / \Lambda_{\chi S B}$, it counts as well the other small scales of the theory, $m_{\pi}$ and $Q^{2}$. Therefore, we shall estimate the error using the following expansion parameter (assuming $\Lambda_{\chi S B} \sim M_{N}$ ):

$$
\tilde{\delta}=\frac{1}{3}\left[\frac{\Delta}{M_{N}}+\left(\frac{m_{\pi}}{M_{N}}\right)^{1 / 2}+\left(\frac{Q^{2}}{M_{N}^{2}}\right)^{1 / 2}\right]
$$


where all the light scales are treated on equal footing and hence are averaged over.

Secondly, what if the amplitude happens to vanish at some kinematical point. According to Eq. (153) the theoretical calculation at that point would be perfect, which is of course unlikely to be true in reality. So, when considering dependencies on kinematical variable(s), we shall take an average of the error over some appropriate region of that variable.

Given these two points, we are led to the following formula for the theoretical uncertainty of the NLO calculation for an amplitude $A$,

$$
A_{e r r}=\left\{\begin{array}{cc}
|A|_{a v} \tilde{\delta}^{2}, & \mathrm{LO} \neq 0 \\
|A|_{a v} \tilde{\delta}, & \mathrm{LO}=0
\end{array}\right.
$$

and the subscript " $a v$ " indicates that the appropriate averaging is performed.

The theoretical uncertainty of the NLO calculation of an observable $O$ is:

$$
O_{e r r}=\left\{\begin{array}{cc}
2|O|_{a v} \tilde{\delta}^{2}, & \mathrm{LO} \neq 0 \\
2|O|_{a v} \tilde{\delta}, & \mathrm{LO}=0,
\end{array}\right.
$$

where the factor of 2 takes into account that an observable is a product of two amplitudes.

Note that this error estimate differs from the one presented in our earlier paper [23], where we overlooked the case of vanishing LO contributions. This underestimated the error in some observables, as will be discussed in more detail below.

\section{RESULTS AND DISCUSSION}

We are now in position to discuss the NLO results for pion photo- and electroproduction amplitudes and observables. We begin with the multipole-analysis for pion photoproduction. The resonant photoproduction multipoles are well-established, and we use them to determine the two low-energy constants: $g_{M}$ and $g_{E}$, the strength of the $M 1$ and $E 2 \gamma N \Delta$ transitions. Subsequently, we discuss the results for pion electroproduction: cross sections, multipoles and the $Q^{2}$ dependence of the $R_{E M}$ and $R_{S M}$ ratios. The comparison with pion electroproduction observables allows to determine the third low energy constant in the $\chi$ EFT framework, related to the strength of the $C 2 \gamma N \Delta$ transition. Once the three low energy constants are determined, the $Q^{2}$ - and $m_{\pi}$-dependencies follow as a prediction of the NLO result. We discuss the predictions for the $m_{\pi}$-dependence of the magnetic dipole $\gamma N \Delta$ form factor, the $R_{E M}$ and $R_{S M}$ ratios, and compare with the recent lattice-QCD results.

\section{A. Pion photoproduction}

In Fig. 7, we show the result of the $\chi \mathrm{EFT}$ calculations for the pion photoproduction resonant multipoles $M_{1+}^{(3 / 2)}$ and $E_{1+}^{(3 / 2)}$, around the resonance position, as function of the total c.m. energy $W$ of the $\pi N$ system. These two multipoles are well established by the MAID [11] and SAID [38] partial-wave solutions which allow us to fit the two low-energy constants of the chiral Lagrangian Eq. (12) as : $g_{M}=2.9, g_{E}=-1.0$. As is seen from the figure, with these values the NLO results (solid lines) give a good description of the energy dependence of the resonant multipoles in a window of $100 \mathrm{MeV}$ around the $\Delta$-resonance position. Also, these values yield $R_{E M}=-2.3 \%$, in a nice agreement with experiment [1]. 
The dashed curves in Fig. [7] show the contribution of the $\Delta$-resonant diagram of Fig. 2(a) without the NLO vertex corrections Fig. 2(e, f). For the $M_{1+}$ multipole this is the LO contribution. For the $E_{1+}$ multipole the LO contribution is absent [recall that the $g_{E}$ coupling is of one order higher than $g_{M}$, see Eq. (12)]. Hence, the dashed curve represents a partial NLO contribution to $E_{1+}$ therein.

Upon adding the non-resonant Born graphs Fig. 2(b) to the dashed curves we obtain the dotted curves in Fig. [7. These non-resonant contributions are purely real at this order and do not affect the imaginary part of the multipoles. One sees that the resulting calculation is flawed because the real parts of the resonant multipoles now fail to cross zero at the resonance position and hence unitarity, in the sense of Watson's theorem [37], is violated. ${ }^{2}$ The complete NLO calculation, shown by the solid curves in the figure includes in addition the vertex corrections Fig. 2(e, f), which restore unitarity exactly. This may come as a surprise, since we are doing a perturbative calculation, without a resummation of rescattering contributions. Nevertheless, it not difficult to see that our numerical result is not a fluke. Watson's theorem is satisfied exactly by the NLO, up to-one-loop amplitude given the graphs in Fig. 2 .

It is also interesting to examine our calculation for the non-resonant multipoles, which all receive contributions of NLO only. In Fig. 8, we show the NLO calculations for the real parts of the non-resonant $s_{-}, p$ - and $d$-wave pion photoproduction multipoles in the $\Delta(1232)$ region in comparison with the two state-of-the-art phenomenological multipole solutions, MAID and SAID. Note that at NLO in the $\delta$-expansion, the non-resonant multipoles are purely real. The multipole solutions show indeed that the imaginary parts of non-resonant multipoles, around the $\Delta$ resonance, are negligibly smaller than their real parts. From the figure one thus sees that for most of the non-resonant multipoles, the parameter-free NLO results, agree fairly well with the phenomenological multipole solutions. The largest differences are observed for $M_{1-}^{(1 / 2) p}$ multipole. This multipole corresponds with nucleon quantum numbers. The cause of the appreciable difference in this channel is largely due to the nucleon anomalous-magnetic-moment contributions, which are not included in our calculation (since they appear at $\mathrm{N}^{2} \mathrm{LO}$ in the $\delta$-expansion), but which are included in the phenomenological solutions.

\section{B. Pion electroproduction}

We now will present the electroproduction observables obtained from the NLO amplitude of Fig. 2] The five-fold pion electroproduction cross section can be expressed as :

$$
\frac{d \sigma}{\left(d E_{e}^{\prime} d \Omega_{e}^{\prime}\right)^{l a b} d \Omega_{\pi}^{c . m .}}=\Gamma_{v} \frac{d \sigma}{d \Omega_{\pi}^{c . m .}}
$$

${ }^{2}$ Recall that Watson's theorem is a simple statement of unitarity based on a coupled-channel scattering equation to leading order in the electromagnetic interaction. The theorem relates the phase of a photoproduction multipole $M_{l}^{(\gamma N)}$ to a corresponding (in spin and parity) $\pi N$-scattering phase-shift $\delta_{l}^{(\pi N)}$ :

$$
M_{l}^{(\gamma N)}=\left|M_{l}^{(\gamma N)}\right| e^{i \delta_{l}^{(\pi N)}} .
$$

For the resonant channel the corresponding phase-shift crosses 90 degrees at the resonance position, and thus the real part of the resonant multipoles must vanish. 
where the virtual photon flux factor $\Gamma_{v}$ is defined as :

$$
\Gamma_{v}=\frac{e^{2}}{(2 \pi)^{3}} \frac{E_{e}^{\prime}}{E_{e}} \frac{\left(W^{2}-M_{N}^{2}\right)}{2 M_{N}} \frac{1}{(1-\varepsilon) Q^{2}},
$$

where $W$ is the invariant mass of the final $\pi N$ system, $E_{e}\left(E_{e}{ }^{\prime}\right)$ are the initial (final) electron $l a b$ energies, and $\varepsilon$ denotes the photon polarization parameter.

The $\gamma^{*} N \rightarrow \pi N$ cross section for unpolarized nucleons is expressed in terms of 5 response functions as :

$$
\begin{aligned}
\frac{d \sigma}{d \Omega_{\pi}} & =\frac{d \sigma_{T}}{d \Omega_{\pi}}+\varepsilon \frac{d \sigma_{L}}{d \Omega_{\pi}}+\varepsilon \cos 2 \Phi \frac{d \sigma_{T T}}{d \Omega_{\pi}} \\
& +\sqrt{2 \varepsilon(1+\varepsilon)} \cos \Phi \frac{d \sigma_{L T}}{d \Omega_{\pi}}+h \sqrt{2 \varepsilon(1-\varepsilon)} \sin \Phi \frac{d \sigma_{L T}^{\prime}}{d \Omega_{\pi}}
\end{aligned}
$$

where $\Theta_{\pi}$ and $\Phi$ are the pion polar and azimuthal c.m. angles, respectively, and $h$ denotes the electron helicity.

In Fig. 9 we show the NLO results for the different virtual photon absorption cross sections entering Eq. (59) at the resonance position, and for $Q^{2} \simeq 0.127 \mathrm{GeV}^{2}$, where recent precision data are available. Besides the low-energy constants $g_{M}$ and $g_{E}$, which were fixed from the resonant multipoles in Fig. [7, the only other low-energy constant from Eq. (12) entering the NLO electroproduction calculation is $g_{C}$. In Eq. (59), the main sensitivity on $g_{C}$ enters in $\sigma_{L T}$. A best description of the $\sigma_{L T}$ data in Fig. 9 is obtained by choosing $g_{C}=-2.36$.

The theoretical uncertainty of the NLO result is estimated by using Eq. (56), where the average is taken over the range of $\Theta_{\pi}$. Note that $\sigma_{L T}$ and $\sigma_{L T^{\prime}}$ do not receive any LO contributions and therefore the $\mathrm{LO}=0$ case in Eq. (56) must be applied in the estimate. This point was overlooked in our first error estimates of the NLO calculation [23], which clearly led to an underestimate of the theory error for $\sigma_{L T}$ and $\sigma_{L T^{\prime}}$.

From Fig. 9, one sees that the NLO $\chi$ EFT calculation, within its accuracy, is consistent with the experimental data for these observables.

The reliability of the present $\chi \mathrm{EFT}$ calculation can also be tested by comparing its predictions for the non-resonant multipoles with the phenomenological multipole solutions MAID and SAID. In Fig. 10, we show this comparison for the non-resonant $s$ - and $p$ wave pion electroproduction multipoles at the resonance position as function of $Q^{2}$. Note that there is a considerable uncertainty in the phenomenologically extracted $s$-wave scalar multipoles $S_{0+}^{(3 / 2)}$ and $S_{0+}^{(1 / 2) p}$ at low $Q^{2}$. As one can see from the figure, our calculation is in a reasonable agreement with the phenomenological solutions for most of the $s$ - and $p$-wave non-resonant multipoles. The largest discrepancy is observed in the $Q^{2}$-dependence of both $E_{0+}^{(3 / 2)}$ and $E_{0+}^{(1 / 2) p}$. This discrepancy will need to be resolved by $s$-wave $\mathrm{N}^{2} \mathrm{LO}$ corrections which grow with $Q^{2}$.

In Fig. 11] we show the $Q^{2}$ dependence of the ratios $R_{E M}$ and $R_{S M}$. Having fixed the low energy constants $g_{M}, g_{E}$ and $g_{C}$, this $Q^{2}$ dependence follows as a prediction. The theoretical uncertainty here (shown by the error bands) is estimated according to Eq. (55), for $\mathrm{LO}=0$ case, and the average taken over the range of $Q^{2}$ from 0 to $0.2 \mathrm{GeV}^{2}$. From the figure one sees that the NLO calculations are consistent with the experimental data for both of the ratios.

To see how higher-order effects may affect the $Q^{2}$ dependence, we include the vectormeson type of dependence for the electric $\gamma N \Delta$ transition by the following replacement of 
the low-energy constant:

$$
g_{E} \rightarrow \frac{g_{E}}{\left(1+Q^{2} / \Lambda_{E}^{2}\right)^{2}}
$$

In contrast to the analogous effect for $g_{M}$, which is of NLO, the inclusion of the $Q^{2}$ dependence in $g_{E}$ is a $\mathrm{N}^{2} \mathrm{LO}$ effect. We choose $\Lambda_{E}$ so as to satisfy the asymptotic condition [42]: $R_{E M} \rightarrow 1$, for $Q^{2} \rightarrow \infty$. This is achieved by the choice $: \Lambda_{E}^{2}=\sqrt{-g_{M}(0) / g_{E}(0)} 0.71 \mathrm{GeV}^{2}$. The dashed curve in Fig. [1] shows the resulting effect of the replacement (601), as imposed on the complete NLO result shown by the solid curves with error bands. The fact that the dashed curves go out of the error bands at some point indicates that our error estimate is not designed for such high $Q^{2}$ values.

\section{Chiral behavior and chiral extrapolations}

Since the low-energy constants $g_{M}, g_{E}$, and $g_{C}$ have been fixed, our calculation can provide a prediction for the $m_{\pi}$ dependence of the $\gamma N \Delta$ transition form factors. The study of the $m_{\pi}$-dependence is crucial to connect to lattice QCD results, which at present can only be obtained for larger pion masses (typically $m_{\pi} \gtrsim 300 \mathrm{MeV}$ ).

The $m_{\pi}$ dependence of the nucleon and $\Delta$-resonance masses, given above by Eq. (19) and (26), are compared with lattice results in Fig. 12] We constrain one of the two parameters in Eq. (19) by the physical nucleon mass value at $m_{\pi}=0.139 \mathrm{GeV}$, while the other parameter is fit to the lattice data shown in the figure. This yields : $M_{N}^{(0)}=0.883 \mathrm{GeV}$ and $c_{1 N}=$ $-0.87 \mathrm{GeV}^{-1}$. As is seen from the figure, with this two-parameter form for $M_{N}$, a good description of lattice results is obtained up to $m_{\pi}^{2} \simeq 0.5 \mathrm{GeV}^{2}$.

Analogously to the nucleon case, we fix one parameter in Eq. (26) from the physical value of the $\Delta$ mass, while the second parameter is fit to the lattice data shown in Fig. 12, yielding : $M_{\Delta}^{(0)}=1.20 \mathrm{GeV}$ and $c_{1 \Delta}=-0.40 \mathrm{GeV}^{-1}$. As well as for the nucleon, this two-parameter form for $M_{\Delta}$ yields a fairly good description of the lattice results up to $m_{\pi}^{2} \simeq 0.5 \mathrm{GeV}^{2}$.

In Fig. 13] we examine the $m_{\pi}$-dependence of the magnetic $\gamma N \Delta$-transition form factor $G_{M}^{*}$, in the convention of Jones and Scadron, see Eq. (37). At the physical pion mass, this

form factor can be obtained from the imaginary part of the $M_{1+}^{3 / 2}$ multipole at $W=M_{\Delta}$ (where the real part is zero by Watson's theorem) as :

$$
\operatorname{Re} G_{M}^{*}\left(Q^{2}\right)=\left(\frac{8 M_{N}^{2}\left|\mathbf{p}_{\pi}^{*}\right| \Gamma_{\Delta}}{3 \alpha_{e m}\left|\mathbf{q}^{*}\right|^{2}}\right)^{1 / 2} \operatorname{Im} M_{1+}^{3 / 2}\left(W=M_{\Delta}, Q^{2}\right),
$$

where $\left|\mathbf{p}_{\pi}^{*}\right|\left(\left|\mathbf{q}_{\pi}^{*}\right|\right)$ denote the pion (virtual photon) c.m. three-momenta respectively at the resonance position, i.e. for $W=M_{\Delta}$. Recall that the value of $G_{M}^{*}$ at $Q^{2}=0$ is determined by the low-energy constant $g_{M}$. The $Q^{2}$-dependence then follows as a prediction of the NLO result, and Fig. 13 shows that this prediction is consistent with the experimental value at $Q^{2}=0.127 \mathrm{GeV}^{2}$ and physical pion mass.

The $m_{\pi^{-}}$-dependence of $G_{M}^{*}$ is also completely fixed at NLO, no new parameters appear. In Fig. [13, the result for $G_{M}^{*}$ at $Q^{2}=0.127 \mathrm{GeV}^{2}$ is shown both when the $m_{\pi}$-dependence of the nucleon and $\Delta$ masses is included and when it is not. Accounting for the $m_{\pi}$-dependence in $M_{N}$ and $M_{\Delta}$, shown in Fig. 12, apparently changes the result for $G_{M}^{*}$ quite significantly. The $\chi$ EFT calculation, with the $m_{\pi}$ dependence of $M_{N}$ and $M_{\Delta}$ included, is in a qualitatively good agreement with the lattice data shown in the figure. The $\chi$ EFT result also follows an 
approximately linear behavior in $m_{\pi}^{2}$, although it falls about $10-15 \%$ below the lattice data. This is just within the uncertainty of the NLO results. One should also keep in mind that the present lattice simulations are not done in full QCD, but are "quenched", so discrepancies are not unexpected.

In Fig. 14, we show the $m_{\pi}$-dependence of the ratios $R_{E M}$ and $R_{S M}$ and compare them to lattice QCD calculations. The recent state-of-the-art lattice calculations of $R_{E M}$ and $R_{S M}$ [25] use a linear, in the quark mass $\left(m_{q} \propto m_{\pi}^{2}\right)$, extrapolation to the physical point, thus assuming that the non-analytic $m_{q}$-dependencies are negligible. The thus obtained value for $R_{S M}$ at the physical $m_{\pi}$ value displays a large discrepancy with the experimental result, as seen in Fig. 14. Our calculation, on the other hand, shows that the non-analytic dependencies are not negligible. While at larger values of $m_{\pi}$, where the $\Delta$ is stable, the ratios display a smooth $m_{\pi}$ dependence, at $m_{\pi}=\Delta$ there is an inflection point, and for $m_{\pi} \leq \Delta$ the non-analytic effects are crucial, as was also observed for the $\Delta$-resonance magnetic moment [24, 44].

One also sees from Fig. 14 that, unlike the result for $G_{M}^{*}$, there is only little difference between the $\chi \mathrm{EFT}$ calculations with the $m_{\pi}$-dependence of $M_{N}$ and $M_{\Delta}$ accounted for, and our earlier calculation [23], where the ratios were evaluated neglecting the $m_{\pi}$-dependence of the masses. This is easily understood, as the main effect due to the $m_{\pi}$-dependence of $M_{N}$ and $M_{\Delta}$ arises due to a common factor in the evaluation of the $\gamma N \Delta$ form factors, which drops out of the ratios. One can speculate that the "quenching" effects drop out, at least partially, from the ratios as well.

In Fig. 14 we also show the $m_{\pi}$-dependence of the $\gamma N \Delta$ transition ratios, with the theoretical uncertainty estimated according to Eq. (55), for the case $\mathrm{LO}=0$, and with the average taken over the range of $m_{\pi}^{2}$ from 0 to $0.15 \mathrm{GeV}^{2}$. The $m_{\pi}$ dependence obtained here from $\chi \mathrm{EFT}$ clearly shows that the lattice results for $R_{S M}$ may in fact be consistent with experiment.

\section{CONCLUSION}

Let us briefly go over the main points and results presented in this paper, which is the first one in a series devoted to $\chi \mathrm{EFT}$ in the $\Delta(1232)$-resonance region.

(i) We develop an extension of chiral perturbation theory to the $\Delta(1232)$-resonance energy region, based on the $\delta$-expansion of Ref. [22]. In this $\chi$ EFT framework the expansion is done in the small parameter $\delta$ equal to the excitation energy of the resonance over the chiral symmetry-breaking scale. The other low-energy scale of the theory, the pion mass, counts as $\delta^{2}$, which is a crucial point for an adequate counting of the $\Delta$-resonance contributions in both the low-energy and the resonance energy regions.

(ii) This framework has been applied here to the process of pion electroproduction. This is a first $\chi \mathrm{EFT}$ study of this reaction in the $\Delta(1232)$-resonance region. We have performed a complete calculation of this process in the resonance region up to, and including, next-to-leading order in the $\delta$-expansion. The power counting in $\delta$ has only been used to establish which graphs contribute at the leading and next-to-leading order, no actual expansion of the diagrams themselves is being done. Therefore, some higher-order in $\delta$ effects, required by relativity and analyticity, are automatically included. Such effects are known to improve the convergence and extend the region of applicability of $\chi$ EFT calculations. 
(iii) Our NLO calculation of pion electroproduction satisfies gauge and chiral symmetries perturbatively, and Lorentz-covariance, analyticity, unitarity (Watson's theorem) exactly.

(iv) The chiral-loop contributions to the $\gamma N \Delta$ transition have been evaluated using two independent techniques: the Feynman parameter method and the sideways dispersion relations. Both methods yield the same result.

(v) The only free parameters entering at this order are the $\gamma N \Delta$ couplings $g_{M}, g_{E}, g_{C}$ characterizing the $M 1, E 2, C 2$ transitions, respectively. By comparing our NLO results with the standard multipole solutions (MAID and SAID) for the photoproduction multipoles we have extracted $g_{M}=2.9$ and $g_{E}=-1.0$, corresponding to $R_{E M}=-2.3 \%$. The NLO $\chi$ EFT result was also found to give a good description of the energy-dependence of most non-resonant $s, p$ and $d$-wave photoproduction multipoles in a $100 \mathrm{MeV}$ window around the $\Delta$-resonance position. From the pion electroproduction cross-section $\sigma_{L T}$ we have extracted $g_{C}=-2.36$, which yields $R_{S M} \simeq-7 \%$ near $Q^{2}=0.1 \mathrm{GeV}^{2}$. In overall, the NLO results are consistent with the experimental data of the recent high-precision measurements at MAMI and BATES.

(vi) The $\chi$ EFT framework plays a dual role in that it allows for an extraction of resonance parameters from observables and predicts their pion-mass dependence. In this way it may provide a crucial connection of present lattice QCD results (obtained at unphysical values of $\left.m_{\pi}\right)$ to the experiment. We have shown here that the opening of the $\Delta \rightarrow \pi N$ decay channel at $m_{\pi}=M_{\Delta}-M_{N}$ induces a pronounced non-analytic behavior of the $R_{E M}$ and $R_{S M}$ ratios. While the linearly-extrapolated lattice QCD results for $R_{S M}$ are in disagreement with experimental data, the $\chi$ EFT prediction of the non-analytic dependencies suggests that these results are in fact consistent with experiment.

(vii) The present calculation is systematically improvable. We have indicated what are the next-next-to-leading order effects, however, at present we could only estimate the theoretical uncertainty of our calculations due to such effects. We have defined and provided a corresponding error band on our NLO results. An actual calculation of $\mathrm{N}^{2} \mathrm{LO}$ effects is a worthwhile topic for a future work.

As high-precision data for low- $Q^{2}$ pion electroproduction in the $\Delta$-resonance region become available from BATES, MAMI and JLab, and the next-generation lattice calculations of the $\gamma N \Delta$ transition are on the way [45], the $\chi$ EFT presented here makes a promise to be the theoretical framework to examine and connect these results.

\section{APPENDIX A: FURTHER EVALUATION OF THE FEYNMAN-PARAMETER INTEGRALS}

To perform the integrals in Eqs. (46a),46c), we first note that $\mathcal{M}_{e}^{2}$ can be written as $\left[\left(x-\beta+y \beta_{\gamma}\right)^{2}-\mathcal{D}^{2}\right]$, with

$$
\mathcal{D}(y)=\left[\left(\beta-y \beta_{\gamma}\right)^{2}-\mu^{2}-\bar{Q}^{2} y(1-y)+i \varepsilon\right]^{1 / 2},
$$


and then use the following elementary integrals

$$
\begin{aligned}
& \int d x \ln \left[(x-a)^{2}-b^{2}\right]=-2 x+(x-a) \ln \left[(x-a)^{2}-b^{2}\right]+b \ln \frac{x-a+b}{x-a-b}+C, \\
& \int d x \frac{1}{(x-a)^{2}-b^{2}}=\frac{1}{2 b} \ln \frac{x-a+b}{x-a-b}+C, \\
& \int d x \frac{x}{(x-a)^{2}-b^{2}}=\frac{1}{2} \ln \left[(x-a)^{2}-b^{2}\right]+\frac{a}{2 b} \ln \frac{x-a+b}{x-a-b}+C, \\
& \int d x \frac{x^{2}}{(x-a)^{2}-b^{2}}=x+a \ln \left[(x-a)^{2}-b^{2}\right]+\frac{a^{2}+b^{2}}{2 b} \ln \frac{x-a+b}{x-a-b}+C,
\end{aligned}
$$

to perform one integration:

$$
\begin{aligned}
g_{M}^{(e)} & =-C_{N \Delta} \int_{0}^{1} d y y \int_{0}^{1-y} d x \ln \mathcal{M}_{e}^{2}=-C_{N \Delta} \int_{0}^{1} d y y\{-2(1-y) \\
& +\left(\alpha-y \alpha_{\gamma}\right) \ln \left[r^{2}(1-y)^{2}+y \mu^{2}\right]+\left(\beta-y \beta_{\gamma}\right) \ln \left[\mu^{2}+\bar{Q}^{2} y(1-y)\right] \\
& \left.+\mathcal{D} \ln \left[\frac{(1-y)\left(\beta-y \beta_{\gamma}-y \bar{Q}^{2}+\mathcal{D}\right)-\mu^{2}+i \varepsilon}{(1-y)\left(\beta-y \beta_{\gamma}-y \bar{Q}^{2}-\mathcal{D}\right)-\mu^{2}+i \varepsilon}\right]\right\}, \\
g_{E}^{(e)} & =C_{N \Delta} \int_{0}^{1} d y y \int_{0}^{1-y} d x\left\{\ln \mathcal{M}_{e}^{2}-2 x[-x+(1-y)(1+r)] \mathcal{M}_{e}^{-2}\right\}=-C_{N \Delta} \int_{0} d y y \\
& \times\left\{\left(\beta-y \beta_{\gamma}-r(1-y)\right) \ln \left[r^{2}(1-y)^{2}+y \mu^{2}\right]+\left(\alpha-y \alpha_{\gamma}+r(1-y)\right) \ln \left[\mu^{2}+\bar{Q}^{2} y(1-y)\right]\right. \\
& \left.=\frac{\beta-y \beta_{\gamma}}{\mathcal{D}}\left(\alpha-y \alpha_{\gamma}+r(1-y)\right) \ln \left[\frac{(1-y)\left(\beta-y \beta_{\gamma}-y \bar{Q}^{2}+\mathcal{D}\right)-\mu^{2}+i \varepsilon}{(1-y)\left(\beta-y \beta_{\gamma}-y \bar{Q}^{2}-\mathcal{D}\right)-\mu^{2}+i \varepsilon}\right]\right\}, \\
g_{C}^{(e)} & =-C_{N \Delta} \int_{0}^{1} d y y(2 y-1) \int_{0}^{1-y} d x[-x+(1-y)(1+r)] \mathcal{M}_{e}^{-2} \\
& =-C_{N \Delta} \int_{0}^{1} d y y(2 y-1) \frac{1}{2}\left\{-\ln \frac{r^{2}(1-y)^{2}+y \mu^{2}}{\mu^{2}+\bar{Q}^{2} y(1-y)}\right. \\
& \left.-\frac{1}{\mathcal{D}}\left(\alpha-y \alpha_{\gamma}+r(1-y)\right) \ln \left[\frac{(1-y)\left(\beta-y \beta_{\gamma}-y \bar{Q}^{2}+\mathcal{D}\right)-\mu^{2}+i \varepsilon}{(1-y)\left(\beta-y \beta_{\gamma}-y \bar{Q}^{2}-\mathcal{D}\right)-\mu^{2}+i \varepsilon}\right]\right\} .
\end{aligned}
$$


Furthermore, define

$$
\begin{aligned}
& \mathcal{I}_{n}=\int_{0}^{1} d y y^{n} \ln \left[(r / \mu)^{2}(1-y)^{2}+y\right], \\
& \mathcal{J}_{n}=\int_{0}^{1} d y y^{n} \ln \left[1+(\bar{Q} / \mu)^{2} y(1-y)\right], \\
& \mathcal{K}_{n}=\int_{0}^{1} d y y^{n} \mathcal{D} \ln \frac{(1-y)\left(\beta-y \beta_{\gamma}-y \bar{Q}^{2}+\mathcal{D}\right)-\mu^{2}}{(1-y)\left(\beta-y \beta_{\gamma}-y \bar{Q}^{2}-\mathcal{D}\right)-\mu^{2}}, \\
& \mathcal{L}_{n}=\int_{0}^{1} d y \frac{y^{n}}{\mathcal{D}} \ln \frac{(1-y)\left(\beta-y \beta_{\gamma}-y \bar{Q}^{2}+\mathcal{D}\right)-\mu^{2}}{(1-y)\left(\beta-y \beta_{\gamma}-y \bar{Q}^{2}-\mathcal{D}\right)-\mu^{2}},
\end{aligned}
$$

Then,

$$
\begin{aligned}
g_{M}^{(e)}= & C_{N \Delta}\left\{\frac{1}{3}(1-\ln \mu)-\alpha \mathcal{I}_{1}+\alpha_{\gamma} \mathcal{I}_{2}-\beta \mathcal{J}_{1}+\beta_{\gamma} \mathcal{J}_{2}-\mathcal{K}_{1}\right\} \\
g_{E}^{(e)}= & -C_{N \Delta}\left\{-\frac{1}{3} \ln \mu-(\beta-r) \mathcal{I}_{1}+\left(\beta_{\gamma}-r\right) \mathcal{I}_{2}-(\alpha+r) \mathcal{J}_{1}+\left(\alpha_{\gamma}+r\right) \mathcal{J}_{2}\right. \\
+ & \left.\beta(\alpha+r) \mathcal{L}_{1}-\left[\beta\left(\alpha_{\gamma}+r\right)+\beta_{\gamma}(\alpha+r)\right] \mathcal{L}_{2}+\beta_{\gamma}\left(\alpha_{\gamma}+r\right) \mathcal{L}_{3}\right\} \\
g_{C}^{(e)}= & \frac{1}{2} C_{N \Delta}\left\{2 \mathcal{I}_{2}-\mathcal{I}_{1}-2 \mathcal{J}_{2}+\mathcal{J}_{1}+(\alpha+r)\left(\mathcal{L}_{1}-2 \mathcal{L}_{2}\right)-\left(\alpha_{\gamma}+r\right)\left(\mathcal{L}_{2}-2 \mathcal{L}_{3}\right)\right\} \\
\mathcal{I}_{1}= & \frac{8-6(\mu / r)^{2}+(\mu / r)^{4}}{2 \sqrt{4(r / \mu)^{2}-1} \arctan \sqrt{4(r / \mu)^{2}-1}} \\
& -\frac{3}{2}+\frac{1}{2}(\mu / r)^{2}+\left[1-2(\mu / r)^{2}+\frac{1}{2}(\mu / r)^{2}\right] \ln (r / \mu) \\
\mathcal{I}_{2}= & \frac{4-\frac{19}{3}(\mu / r)^{2}+\frac{8}{3}(\mu / r)^{4}-\frac{1}{3}(\mu / r)^{6}}{\sqrt{4(r / \mu)^{2}-1}} \arctan \sqrt{4(r / \mu)^{2}-1} \\
& -\frac{11}{9}+\frac{3}{2}(\mu / r)^{2}-\frac{1}{3}(\mu / r)^{4}+\left[\frac{2}{3}-3(\mu / r)^{2}+2(\mu / r)^{4}\right] \ln (r / \mu) \\
\mathcal{J}_{1}= & -1+\frac{\sqrt{4 \mu^{2}+\bar{Q}^{2}}}{2 \bar{Q}} \ln \frac{\sqrt{4 \mu^{2}+\bar{Q}^{2}}+\bar{Q}}{\sqrt{4 \mu^{2}+\bar{Q}^{2}}-\bar{Q}} \\
\mathcal{J}_{2}= & -\frac{13}{18}-\frac{2 \mu^{2}}{3 \bar{Q}^{2}}+\frac{2\left(\frac{\bar{Q}}{\mu}+\frac{5 \mu}{Q}+\frac{4 \mu^{3}}{\bar{Q}^{3}}\right)}{3 \sqrt{4 \mu^{2}+\bar{Q}^{2}}} \ln \frac{\sqrt{4 \mu^{2}+\bar{Q}^{2}}+\bar{Q}}{\sqrt{4 \mu^{2}+\bar{Q}^{2}}-\bar{Q}}
\end{aligned}
$$

\section{APPENDIX B: ABSORPTIVE PARTS OF THE VERTEX CORRECTIONS}

In the sideways dispersion relation method, we evaluate the $\pi N$ loop contribution to the $\gamma N \Delta$ form factors using a dispersion relation in the invariant mass of the $\Delta$. To present the results of this technique, we first introduce a number of kinematical quantities and notations. The pion momentum in the rest frame of the decaying $\Delta$ with invariant mass $W=\sqrt{s}$ is given by $\left|\mathbf{p}_{\pi}\right|=W \tilde{\lambda}$, with

$$
\begin{aligned}
\tilde{\lambda} & =\frac{1}{2} \sqrt{\left[1-(\tilde{r}+\tilde{\mu})^{2}\right]\left[1-(\tilde{r}-\tilde{\mu})^{2}\right]} \\
& =\frac{1}{2} \sqrt{\left[(1+\tilde{r})^{2}-\tilde{\mu}^{2}\right]\left[(1-\tilde{r})^{2}-\tilde{\mu}^{2}\right]}
\end{aligned}
$$


and $\tilde{\mu}=m_{\pi} / W, \tilde{r}=M_{N} / W$. We also introduce $r_{\Delta}=M_{\Delta} / W$. In the on-shell case, $W=M_{\Delta}$, we of course have $\tilde{r}=r, \tilde{\mu}=\mu$, and $\tilde{\lambda}=\lambda$.

Furthermore, the energies of the pion $\left(E_{\pi}\right)$ and nucleon $\left(E_{N}\right)$ in the rest frame of the decaying $\Delta$ can be expressed as $E_{\pi}=W \tilde{\beta}$ and $E_{N}=W \tilde{\alpha}$ with the fractions given by:

$$
\tilde{\beta}=\frac{1}{2}\left(1-\tilde{r}^{2}+\tilde{\mu}^{2}\right), \quad \tilde{\alpha}=\frac{1}{2}\left(1+\tilde{r}^{2}-\tilde{\mu}^{2}\right)=1-\tilde{\beta} .
$$

and the Lorentz-invariant pion (nucleon) velocities, denoted by $v_{\pi}\left(v_{N}\right)$ respectively are given by:

$$
v_{\pi} \equiv \frac{\left|\mathbf{p}_{\pi}\right|}{E_{\pi}}=\frac{\tilde{\lambda}}{\tilde{\beta}}, \quad v_{N} \equiv \frac{\left|\mathbf{p}_{N}\right|}{E_{N}}=\frac{\tilde{\lambda}}{\tilde{\alpha}}
$$

where we used $\left|\mathbf{p}_{N}\right|=\left|\mathbf{p}_{\pi}\right|$ in the $\Delta$ rest frame.

We denote the virtual photon four-momentum by $q=\left(q^{0}, \mathbf{q}\right)$. For spacelike virtual photons, the virtuality is usually denoted by $Q^{2} \equiv \mathbf{q}^{2}-q_{0}^{2}>0$. The virtual photon threemomentum in the $\Delta$ rest frame can then be expressed as $|\mathbf{q}|=W \tilde{\kappa}$, with

$$
\begin{aligned}
\tilde{\kappa} & =\frac{1}{2} \sqrt{\left(1-\tilde{r}^{2}\right)^{2}+2 \tilde{Q}^{2}\left(1+\tilde{r}^{2}\right)+\tilde{Q}^{4}}, \\
& =\frac{1}{2} \sqrt{\left[(1+\tilde{r})^{2}+\tilde{Q}^{2}\right]\left[(1-\tilde{r})^{2}+\tilde{Q}^{2}\right]},
\end{aligned}
$$

where $\tilde{Q}^{2} \equiv Q^{2} / s$. It is also useful to introduce the fraction of the photon energy:

$$
\tilde{\beta}_{\gamma} \equiv \frac{p^{\prime} \cdot q}{s}=\frac{1}{2}\left(1-\tilde{r}^{2}-\tilde{Q}^{2}\right)
$$

satisfying $\kappa^{2}=\tilde{\beta}_{\gamma}^{2}+\tilde{Q}^{2}$. Finally, we define

$$
\begin{gathered}
w_{\pi} \equiv \frac{2\left|\mathbf{p}_{\pi}\right||\mathbf{q}|}{Q^{2}+2 E_{\pi} q^{0}}=\frac{2 \tilde{\kappa} \tilde{\lambda}}{\tilde{Q}^{2}+2 \tilde{\beta} \tilde{\beta}_{\gamma}} \\
w_{N} \equiv \frac{2\left|\mathbf{p}_{N}\right||\mathbf{q}|}{Q^{2}+2 E_{N} q^{0}}=\frac{2 \tilde{\kappa} \tilde{\lambda}}{\tilde{Q}^{2}+2 \tilde{\alpha} \tilde{\beta}_{\gamma}} .
\end{gathered}
$$

In the real photon limit $\left(Q^{2} \rightarrow 0\right)$, one obtains that $w_{\pi} \rightarrow v_{\pi}$, and $w_{N} \rightarrow v_{N}$.

The calculation of the absorptive part of these $\pi N$ loop contribution, entering the sideways dispersion relations, involves several phase space integrals, of which the simplest is of the form :

$$
\int \frac{d^{4} p_{\pi}}{(2 \pi)^{4}}\left[(2 \pi) \delta\left(p_{\pi}^{2}-m_{\pi}^{2}\right) \Theta\left(p_{\pi}^{0}\right)\right]\left[(2 \pi) \delta\left(\left(p^{\prime}-p_{\pi}\right)^{2}-M_{N}^{2}\right) \Theta\left(p^{\prime 0}-p_{\pi}^{0}\right)\right]=\frac{\tilde{\lambda}}{4 \pi}
$$

The evaluation of the absorptive part corresponding with Fig. 5 (a), firstly involves the following phase space integrals with different powers of pion momentum in the numerator :

$$
\begin{aligned}
& \int \frac{d^{4} p_{\pi}}{(2 \pi)^{4}}\left[(2 \pi) \delta\left(p_{\pi}^{2}-m_{\pi}^{2}\right) \Theta\left(p_{\pi}^{0}\right)\right]\left[(2 \pi) \delta\left(\left(p^{\prime}-p_{\pi}\right)^{2}-M_{N}^{2}\right) \Theta\left(p^{\prime 0}-p_{\pi}^{0}\right)\right] p_{\pi}^{\alpha} \\
& =a_{1, \pi} p^{\prime \alpha} \\
& \int \frac{d^{4} p_{\pi}}{(2 \pi)^{4}}\left[(2 \pi) \delta\left(p_{\pi}^{2}-m_{\pi}^{2}\right) \Theta\left(p_{\pi}^{0}\right)\right]\left[(2 \pi) \delta\left(\left(p^{\prime}-p_{\pi}\right)^{2}-M_{N}^{2}\right) \Theta\left(p^{\prime 0}-p_{\pi}^{0}\right)\right] p_{\pi}^{\alpha} p_{\pi}^{\beta} \\
& =a_{2, \pi} p^{\prime 2} g^{\alpha \beta}+a_{3, \pi} p^{\prime \alpha} p^{\prime \beta},
\end{aligned}
$$


with

$$
\begin{aligned}
& a_{1, \pi}=\frac{\tilde{\lambda}}{4 \pi} \tilde{\beta}, \\
& a_{2, \pi}=\frac{1}{4 \pi}\left(-\frac{\tilde{\lambda}^{3}}{3}\right), \\
& a_{3, \pi}=\frac{1}{4 \pi} \tilde{\lambda}\left(\tilde{\beta}^{2}+\frac{\tilde{\lambda}^{2}}{3}\right) .
\end{aligned}
$$

Furthermore, the calculation of the absorptive part corresponding with Fig. [5 (a), which has one pion which is off-shell, also involves the following integrals :

$$
\begin{aligned}
& \int \frac{d^{4} p_{\pi}}{(2 \pi)^{4}}\left[(2 \pi) \delta\left(p_{\pi}^{2}-m_{\pi}^{2}\right) \Theta\left(p_{\pi}^{0}\right)\right]\left[(2 \pi) \delta\left(\left(p^{\prime}-p_{\pi}\right)^{2}-M_{N}^{2}\right) \Theta\left(p^{\prime 0}-p_{\pi}^{0}\right)\right] \frac{1}{q^{2}-2 p_{\pi} \cdot q} \\
& =-\frac{1}{2 p^{\prime 2}} b_{1, \pi}, \\
& \int \frac{d^{4} p_{\pi}}{(2 \pi)^{4}}\left[(2 \pi) \delta\left(p_{\pi}^{2}-m_{\pi}^{2}\right) \Theta\left(p_{\pi}^{0}\right)\right]\left[(2 \pi) \delta\left(\left(p^{\prime}-p_{\pi}\right)^{2}-M_{N}^{2}\right) \Theta\left(p^{\prime 0}-p_{\pi}^{0}\right)\right] \frac{p_{\pi}^{\alpha}}{q^{2}-2 p_{\pi} \cdot q} \\
& =-\frac{1}{2 p^{\prime 2}}\left\{b_{2, \pi} p^{\prime \alpha}+b_{3, \pi} q^{\alpha}\right\}, \\
& \int \frac{d^{4} p_{\pi}}{(2 \pi)^{4}}\left[(2 \pi) \delta\left(p_{\pi}^{2}-m_{\pi}^{2}\right) \Theta\left(p_{\pi}^{0}\right)\right]\left[(2 \pi) \delta\left(\left(p^{\prime}-p_{\pi}\right)^{2}-M_{N}^{2}\right) \Theta\left(p^{\prime 0}-p_{\pi}^{0}\right)\right] \frac{p_{\pi}^{\alpha} p_{\pi}^{\beta}}{q^{2}-2 p_{\pi} \cdot q} \\
& =-\frac{1}{2}\left\{c_{1, \pi} g^{\alpha \beta}+c_{1, \pi}^{\prime}\left(\frac{q^{\alpha} p^{\prime \beta}+q^{\beta} p^{\prime \alpha}}{q \cdot p^{\prime}}\right)+\frac{c_{2, \pi}}{p^{\prime 2}} p^{\prime \alpha} p^{\prime \beta}+\frac{c_{3, \pi}}{p^{\prime 2}} q^{\alpha} q^{\beta}\right\}, \\
& \int \frac{d^{4} p_{\pi}}{(2 \pi)^{4}}\left[(2 \pi) \delta\left(p_{\pi}^{2}-m_{\pi}^{2}\right) \Theta\left(p_{\pi}^{0}\right)\right]\left[(2 \pi) \delta\left(\left(p^{\prime}-p_{\pi}\right)^{2}-M_{N}^{2}\right) \Theta\left(p^{\prime 0}-p_{\pi}^{0}\right)\right] \frac{p_{\pi}^{\alpha} p_{\pi}^{\beta} p_{\pi}^{\gamma}}{q^{2}-2 p_{\pi} \cdot q} \\
& =-\frac{1}{2}\left\{d_{1, \pi}\left[g^{\alpha \beta} p^{\prime \gamma}+g^{\beta \gamma} p^{\prime \alpha}+g^{\gamma \alpha} p^{\prime \beta}\right]\right. \\
& +d_{1, \pi}^{\prime} \frac{2}{q \cdot p^{\prime}}\left[q^{\alpha} p^{\prime \beta} p^{\prime \gamma}+q^{\beta} p^{\prime \alpha} p^{\prime \gamma}++q^{\gamma} p^{\prime \alpha} p^{\prime \beta}\right] \\
& +d_{2, \pi}\left(g^{\alpha \beta} q^{\gamma}+g^{\beta \gamma} q^{\alpha}+g^{\gamma \alpha} q^{\beta}\right) \\
& +d_{2, \pi}^{\prime} \frac{2}{q \cdot p^{\prime}}\left[q^{\alpha} q^{\beta} p^{\prime \gamma}+q^{\beta} q^{\gamma} p^{\prime \alpha}+q^{\gamma} q^{\alpha} p^{\prime \beta}\right] \\
& \left.+\frac{d_{3, \pi}}{p^{\prime 2}} p^{\prime \alpha} p^{\prime \beta} p^{\prime \gamma}+\frac{d_{4, \pi}}{p^{\prime 2}} q^{\alpha} q^{\beta} q^{\gamma}\right\} \text {. }
\end{aligned}
$$


The coefficients appearing in the above equations are given by :

$$
\begin{aligned}
& b_{1, \pi}=\frac{1}{4 \pi} \frac{1}{2 \tilde{\kappa}} \log \left(\frac{1+w_{\pi}}{1-w_{\pi}}\right) \text {, } \\
& b_{2, \pi}=\frac{1}{4 \pi} \frac{1}{4 \tilde{\kappa}^{2}}\left\{4 \tilde{\lambda} \tilde{\beta}_{\gamma}+\tilde{Q}^{2}\left(2 \tilde{\beta}-\tilde{\beta}_{\gamma}\right) \frac{1}{\tilde{\kappa}} \log \left(\frac{1+w_{\pi}}{1-w_{\pi}}\right)\right\} \text {, } \\
& b_{3, \pi}=\frac{1}{4 \pi} \frac{\tilde{\lambda}}{2 \tilde{\kappa}^{2}}\left\{-2+\frac{1}{w_{\pi}} \log \left(\frac{1+w_{\pi}}{1-w_{\pi}}\right)\right\} \text {, } \\
& c_{1, \pi}=\frac{1}{4 \pi} \frac{\tilde{\lambda}^{2}}{4 \tilde{\kappa}}\left\{-\frac{2}{w_{\pi}}+\log \left(\frac{1+w_{\pi}}{1-w_{\pi}}\right)\left[-1+\frac{1}{w_{\pi}^{2}}\right]\right\}, \\
& c_{1, \pi}^{\prime}=\frac{1}{4 \pi} \frac{1}{4 \tilde{\kappa}^{2}}\left\{\tilde{\lambda}\left[\left(1-\frac{3 \tilde{Q}^{2}}{\tilde{\kappa}^{2}}\right)\left(2 \tilde{\beta} \tilde{\beta}_{\gamma}+\tilde{Q}^{2}\right)+2 \tilde{Q}^{2}\right]\right. \\
& \left.+\frac{1}{2 \tilde{\kappa}} \log \left(\frac{1+w_{\pi}}{1-w_{\pi}}\right)\left[\tilde{\lambda}^{2}\left(2 \tilde{\beta}_{\gamma}^{2}\left(1-\frac{1}{w_{\pi}^{2}}\right)+\frac{4 \tilde{Q}^{2}}{w_{\pi}^{2}}\right)-\tilde{Q}^{2}\left(2 \tilde{\beta} \tilde{\beta}_{\gamma}+\tilde{Q}^{2}\right)\right]\right\}, \\
& c_{2, \pi}=\frac{1}{4 \pi} \frac{1}{4 \tilde{\kappa}^{2}}\left\{\tilde{\lambda}\left[\left(1+\frac{3 \tilde{Q}^{2}}{2 \tilde{\kappa}^{2}}\right) 2\left(2 \tilde{\beta} \tilde{\beta}_{\gamma}+\tilde{Q}^{2}\right)-4 \tilde{Q}^{2}\right]\right. \\
& \left.+\frac{\tilde{Q}^{2}}{2 \tilde{\kappa}} \log \left(\frac{1+w_{\pi}}{1-w_{\pi}}\right)\left[2 \tilde{\lambda}^{2}\left(1-\frac{3}{w_{\pi}^{2}}\right)+4 \tilde{\beta}^{2}+\tilde{Q}^{2}\right]\right\} \\
& c_{3, \pi}=\frac{1}{4 \pi} \frac{\tilde{\lambda}^{2}}{4 \tilde{\kappa}^{3}}\left\{-\frac{6}{w_{\pi}}+\log \left(\frac{1+w_{\pi}}{1-w_{\pi}}\right)\left[-1+\frac{3}{w_{\pi}^{2}}\right]\right\} \text {, } \\
& d_{1, \pi}=\frac{1}{4 \pi} \frac{\tilde{\lambda}^{2}}{4 \tilde{\kappa}^{3}}\left\{-\frac{4}{3} \tilde{\kappa} \tilde{\lambda} \tilde{\beta}_{\gamma}-\frac{\tilde{Q}^{2}\left(2 \tilde{\beta}-\tilde{\beta}_{\gamma}\right)}{w_{\pi}}+\frac{\tilde{Q}^{2}}{2} \log \left(\frac{1+w_{\pi}}{1-w_{\pi}}\right)\left(2 \tilde{\beta}-\tilde{\beta}_{\gamma}\right)\left[-1+\frac{1}{w_{\pi}^{2}}\right]\right\}, \\
& d_{1, \pi}^{\prime}=\frac{1}{4 \pi} \frac{\tilde{\beta}_{\gamma}}{8 \tilde{\kappa}^{4}}\left\{\tilde{\lambda}\left[2 \tilde{\lambda}^{2}\left(\frac{2}{3}\left(\tilde{\beta}_{\gamma}^{2}-\tilde{Q}^{2}\right)+\frac{5 \tilde{Q}^{2}}{w_{\pi}^{2}}\right)-\tilde{Q}^{2}\left(4 \tilde{\beta}^{2}+2 \tilde{Q}^{2}+2 \tilde{\beta} \tilde{\beta}_{\gamma}\right)\right]\right. \\
& +\frac{\tilde{Q}^{2}}{2 \tilde{\kappa}} \log \left(\frac{1+w_{\pi}}{1-w_{\pi}}\right)\left[\tilde{\lambda}^{2}\left(\tilde{\beta}_{\gamma}\left(2 \tilde{\beta}-\tilde{\beta}_{\gamma}\right)\left(3-\frac{5}{w_{\pi}^{2}}\right)+\tilde{\kappa}^{2}\left(1-\frac{1}{w_{\pi}^{2}}\right)\right)\right. \\
& \left.\left.+\tilde{\beta}\left(2 \tilde{\beta}-\tilde{\beta}_{\gamma}\right)\left(2 \tilde{\beta} \tilde{\beta}_{\gamma}+\tilde{Q}^{2}\right)\right]\right\},
\end{aligned}
$$




$$
\begin{aligned}
d_{2, \pi} & =\frac{1}{4 \pi} \frac{\tilde{\lambda}^{3}}{2 \tilde{\kappa}^{2}}\left\{\frac{2}{3}-\frac{1}{w_{\pi}^{2}}+\frac{1}{2 w_{\pi}} \log \left(\frac{1+w_{\pi}}{1-w_{\pi}}\right)\left[-1+\frac{1}{w_{\pi}^{2}}\right]\right\} \\
d_{2, \pi}^{\prime} & =\frac{1}{4 \pi} \frac{1}{8 \tilde{\kappa}^{4}}\left\{\tilde{\lambda}\left[2 \tilde{\lambda}^{2}\left(\tilde{\beta}_{\gamma}^{2}\left(-\frac{4}{3}+\frac{2}{w_{\pi}^{2}}\right)-\frac{3 \tilde{Q}^{2}}{w_{\pi}^{2}}\right)+\frac{3}{2} \tilde{Q}^{2}\left(2 \tilde{\beta} \tilde{\beta}_{\gamma}+\tilde{Q}^{2}\right)\right]\right. \\
& \left.+\frac{\tilde{\lambda}^{2}}{2 \tilde{\kappa}} \log \left(\frac{1+w_{\pi}}{1-w_{\pi}}\right)\left[4 \tilde{\kappa}^{2} \tilde{\beta} \tilde{\beta}_{\gamma}\left(1-\frac{1}{w_{\pi}^{2}}\right)-\tilde{\beta}_{\gamma} \tilde{Q}^{2}\left(2 \tilde{\beta}-\tilde{\beta}_{\gamma}\right)\left(3-\frac{5}{w_{\pi}^{2}}\right)\right]\right\} \\
d_{3, \pi} & =-3 d_{1, \pi}-4 d_{1, \pi}^{\prime}-2 \tilde{\beta}_{\gamma}\left(d_{2, \pi}+d_{2, \pi}^{\prime}\right)+\tilde{\beta}^{2} b_{2, \pi}, \\
d_{4, \pi} & =\frac{2}{\tilde{Q}^{2}}\left(d_{2, \pi}+d_{2, \pi}^{\prime}\right)+\frac{1}{2} c_{3, \pi} .
\end{aligned}
$$

The calculation of the absorptive part corresponding with Fig. [5 (b), which has one nucleon which is off-shell, involves the integrals :

$$
\begin{aligned}
& \int \frac{d^{4} p_{N}}{(2 \pi)^{4}}\left[(2 \pi) \delta\left(p_{N}^{2}-M_{N}^{2}\right) \Theta\left(p_{N}^{0}\right)\right]\left[(2 \pi) \delta\left(\left(p^{\prime}-p_{N}\right)^{2}-m_{\pi}^{2}\right) \Theta\left(p^{\prime 0}-p_{N}^{0}\right)\right] p_{N}^{\alpha} \\
& =a_{1, N} p^{\alpha}, \\
& \int \frac{d^{4} p_{N}}{(2 \pi)^{4}}\left[(2 \pi) \delta\left(p_{N}^{2}-M_{N}^{2}\right) \Theta\left(p_{N}^{0}\right)\right]\left[(2 \pi) \delta\left(\left(p^{\prime}-p_{N}\right)^{2}-m_{\pi}^{2}\right) \Theta\left(p^{\prime 0}-p_{N}^{0}\right)\right] p_{N}^{\alpha} p_{N}^{\beta} \\
& =a_{2, N} p^{\prime 2} g^{\alpha \beta}+a_{3, N} p^{\prime \alpha} p^{\prime \beta}, \\
& \int \frac{d^{4} p_{N}}{(2 \pi)^{4}}\left[(2 \pi) \delta\left(p_{N}^{2}-M_{N}^{2}\right) \Theta\left(p_{N}^{0}\right)\right]\left[(2 \pi) \delta\left(\left(p^{\prime}-p_{N}\right)^{2}-m_{\pi}^{2}\right) \Theta\left(p^{\prime 0}-p_{N}^{0}\right)\right] \frac{1}{q^{2}-2 p_{N} \cdot q} \\
& =-\frac{1}{2 p^{\prime 2}} b_{1, N},
\end{aligned}
$$

and analogous integrals as in Eqs. (B15,B17), where $p_{\pi} \leftrightarrow p_{N}$.

The coefficients appearing in the above integrals are given by :

$$
\begin{aligned}
& a_{1, N}=\frac{\tilde{\lambda}}{4 \pi} \tilde{\alpha} \\
& a_{2, N}=\frac{1}{4 \pi}\left(-\frac{\tilde{\lambda}^{3}}{3}\right), \\
& a_{3, N}=\frac{1}{4 \pi} \tilde{\lambda}\left(\tilde{r}^{2}+\frac{4 \tilde{\lambda}^{2}}{3}\right) .
\end{aligned}
$$

and

$$
\begin{aligned}
& b_{1, N}=\frac{1}{4 \pi} \frac{1}{2 \tilde{\kappa}} \log \left(\frac{1+w_{N}}{1-w_{N}}\right) \\
& b_{2, N}=\frac{1}{4 \pi} \frac{1}{4 \tilde{\kappa}^{2}}\left\{4 \tilde{\lambda} \tilde{\beta}_{\gamma}+\tilde{Q}^{2}\left[1+\tilde{r}^{2}+\tilde{Q}^{2}+2\left(\tilde{r}^{2}-\tilde{\mu}^{2}\right)\right] \frac{1}{2 \tilde{\kappa}} \log \left(\frac{1+w_{N}}{1-w_{N}}\right)\right\}, \\
& b_{3, N}=\frac{1}{4 \pi} \frac{1}{2 \tilde{\kappa}^{2}}\left\{-2 \tilde{\lambda}+\left(2 \tilde{\alpha} \tilde{\beta}_{\gamma}+\tilde{Q}^{2}\right) \frac{1}{2 \tilde{\kappa}} \log \left(\frac{1+w_{N}}{1-w_{N}}\right)\right\}
\end{aligned}
$$




$$
\begin{aligned}
& c_{1, N}= \frac{1}{4 \tilde{\kappa}^{2}}\left\{-2 \tilde{\beta}_{\gamma} a_{1, N}+2\left(\tilde{\beta}_{\gamma}^{2} \tilde{r}^{2}-\tilde{Q}^{2} \tilde{\lambda}^{2}\right) b_{1, N}+\tilde{Q}^{2} \tilde{\beta}_{\gamma} b_{2, N}+\tilde{Q}^{2}\left(\tilde{\kappa}^{2}+\tilde{\beta}_{\gamma}^{2}\right) b_{3, N}\right\} \\
& c_{1, N}^{\prime}=-\left(1-\frac{3 \tilde{Q}^{2}}{\tilde{\kappa}^{2}}\right) c_{1, N}+\tilde{Q}^{2} \frac{\tilde{\lambda}^{2}}{\tilde{\kappa}^{2}} b_{1, N}-\frac{\tilde{Q}^{2}}{2} b_{3, N} \\
& c_{2, N}=-2 c_{1, N}-\tilde{Q}^{2} c_{3, N}+\tilde{r}^{2} b_{1, N}+\tilde{Q}^{2} b_{3, N} \\
& c_{3, N}=\frac{1}{\tilde{\kappa}^{2}}\left\{3 c_{1, N}+\tilde{\lambda}^{2} b_{1, N}\right\} \\
& d_{1, N}=\frac{1}{2 \tilde{\kappa}^{2}}\left\{-\tilde{\beta}_{\gamma}\left(2 a_{2, N}+a_{3, N}\right)+\left[\tilde{r}^{2}\left(\tilde{\kappa}^{2}-\tilde{Q}^{2}\right)-\tilde{Q}^{2} \tilde{\lambda}^{2}\right] b_{2, N}\right. \\
&\left.\quad+\frac{\tilde{Q}^{2}}{2 \tilde{\beta}_{\gamma}}\left[2 \tilde{\kappa}^{2}-\tilde{Q}^{2}\right] c_{1, N}^{\prime}+\frac{\tilde{Q}^{2}}{2} \tilde{\beta}_{\gamma}\left(c_{2, N}+2 c_{1, N}\right)\right\} \\
& d_{1, N}^{\prime}=-\frac{5}{2} d_{1, N}+\frac{\tilde{r}^{2}}{2} b_{2, N}-\frac{\left(1-\tilde{r}^{2}\right)}{4 \tilde{\kappa}^{2}} a_{3, N} \\
& \quad+\frac{\tilde{Q}^{2}}{2 \tilde{\kappa}^{2}}\left[5 d_{1, N}+\frac{1}{2} a_{3, N}-\tilde{r}^{2} b_{2, N}+\frac{1}{2} \tilde{\beta}_{\gamma}\left(c_{1, N}^{\prime}+c_{2, N}\right)\right] \\
& d_{2, N}=\frac{1}{\tilde{Q}^{2}} \tilde{\beta}_{\gamma} d_{1, N}+\frac{1}{2} c_{1, N}-\frac{1}{\tilde{Q}^{2}} a_{2, N} \\
& d_{2, N}^{\prime}=\frac{1}{2 \tilde{Q}^{2}} \tilde{\beta}_{\gamma}\left(d_{1, N}+2 d_{1, N}^{\prime}\right)+\frac{1}{4} c_{1, N}^{\prime} \\
& d_{3, N}=-3 d_{1, N}-4 d_{1, N}^{\prime}-2 \tilde{\beta}_{\gamma}\left(d_{2, N}+d_{2, N}^{\prime}\right)+\tilde{\alpha}^{2} b_{2, N} \\
& d_{4, N}=\frac{2}{\tilde{Q}^{2}}\left(d_{2, N}+d_{2, N}^{\prime}\right)+\frac{1}{2} c_{3, N} \\
& \\
&=
\end{aligned}
$$

The imaginary parts of $g_{1}, g_{2}$, and $g_{3}$ corresponding with the diagram of Fig. 5 (a) are thus given by:

$$
\begin{aligned}
\operatorname{Im} g_{1, a}^{+} & =\frac{g_{A} h_{A} M_{N}}{4 f_{\pi}^{2}}\left\{d_{2, \pi}\right\}, \\
\operatorname{Im} g_{2, a}^{+} & =-\frac{g_{A} h_{A} M_{N}}{4 f_{\pi}^{2}} \frac{1}{W}\left\{\frac{\left(r_{\Delta}+\tilde{r}\right)}{\tilde{\beta}_{\gamma}}\left(2 d_{2, \pi}^{\prime}-c_{1, \pi}^{\prime}\right)+\frac{2 r_{\Delta}}{\tilde{\beta}_{\gamma}} d_{1, \pi}^{\prime}\right\}, \\
\operatorname{Im} g_{3, a}^{+} & =\frac{g_{A} h_{A} M_{N}}{4 f_{\pi}^{2}} \frac{1}{W}\left\{\frac{\left(r_{\Delta}+\tilde{r}\right)}{2}\left(b_{3, \pi}-3 c_{3, \pi}+2 d_{4, \pi}\right)+\frac{r_{\Delta}}{2 \tilde{\beta}_{\gamma}}\left(4 d_{2, \pi}^{\prime}-c_{1, \pi}^{\prime}\right)\right\} .
\end{aligned}
$$

The imaginary parts of $g_{1}, g_{2}$, and $g_{3}$ corresponding with the diagram of Fig. [5 (b) are likewise given by:

$$
\begin{aligned}
& \operatorname{Im} g_{1, b}^{+}=-\frac{g_{A} h_{A} M_{N}}{4 f_{\pi}^{2}}\left\{d_{2 N}+\frac{1}{2} \tilde{r}\left(r_{\Delta}-\tilde{r}\right) b_{3, N}+\frac{\left(1-\tilde{r} r_{\Delta}\right)}{2 \tilde{\beta}_{\gamma}} c_{1, N}^{\prime}-\frac{\tilde{Q}^{2}}{2} c_{3, N}\right\}, \\
& \operatorname{Im} g_{2, b}^{+}=\frac{g_{A} h_{A} M_{N}}{4 f_{\pi}^{2} W}\left\{-\tilde{r} b_{3, N}-\frac{\left(r_{\Delta}-\tilde{r}\right)}{\tilde{\beta}_{\gamma}} c_{1, N}^{\prime}+\frac{2 r_{\Delta}}{\tilde{\beta}_{\gamma}} d_{1, N}^{\prime}+\frac{2\left(r_{\Delta}+\tilde{r}\right)}{\tilde{\beta}_{\gamma}} d_{2, N}^{\prime}\right\}, \\
& \operatorname{Im} g_{3, b}^{+}=\frac{g_{A} h_{A} M_{N}}{4 f_{\pi}^{2}} \frac{1}{W}\left\{r_{\Delta} c_{3, N}-\frac{2 r_{\Delta}}{\tilde{\beta}_{\gamma}} d_{2, N}^{\prime}-\left(r_{\Delta}+\tilde{r}\right) d_{4, N}\right\} .
\end{aligned}
$$




\section{ACKNOWLEDGMENTS}

We thank C. Alexandrou for helpful discussions of the lattice QCD results, and C.-W. Kao for drawing our attention to Ref. [46]. This work is supported in part by DOE grant no. DE-FG02-04ER41302 and contract DE-AC05-84ER-40150 under which SURA operates the Jefferson Laboratory.

[1] R. Beck et al., Phys. Rev. Lett. 78, 606 (1997); Phys. Rev. C 61, 035204 (2000).

[2] G. Blanpied et al., Phys. Rev. Lett. 79, 4337 (1997).

[3] C. Mertz et al., Phys. Rev. Lett. 86, 2963 (2001); N. F. Sparveris et al., ibid. 94, 022003 (2005).

[4] V. V. Frolov et al., Phys. Rev. Lett. 82, 45 (1999); K. Joo et al., ibid. 88, 122001 (2002).

[5] S. L. Glashow, Physica 96A, 27 (1979); N. Isgur, G. Karl and R. Koniuk, Phys. Rev. D 25, 2394 (1982); S. Capstick and G. Karl, ibid. 41, 2767 (1990); S. Capstick and B. D. Kiester, ibid. 51, 3598 (1995); G. A. Miller, Phys. Rev. C 68, 022201(R) (2003); A. M. Bernstein, Eur. Phys. J. A 17, 349 (2003).

[6] M.G. Olsson and E.T. Osypowski, Nucl. Phys. B87, 399 (1974); Phys. Rev. D 17, 174 (1978); R. M. Davidson, N. C. Mukhopadhyay and R. S. Wittman, ibid. 43, 71 (1991).

[7] H. Garcilazo and E. Moya de Guerra, Nucl. Phys. A 562, 521 (1993); C. Fernandez-Ramirez, E. Moya de Guerra and J. M. Udias, "Effective Lagrangian approach to pion photoproduction from the nucleon," arXiv:nucl-th/0509020.

[8] M. Vanderhaeghen, K. Heyde, J. Ryckebusch and M. Waroquier, Nucl. Phys. A 595, 219 (1995); D. Drechsel, M. Vanderhaeghen, M. M. Giannini and E. Santopinto, Phys. Lett. B 484, 236 (2000); D. Drechsel and M. Vanderhaeghen, Phys. Rev. C 64, 065202 (2001).

[9] V. Pascalutsa and O. Scholten, Nucl. Phys. A 591, 658 (1995); O. Scholten, A. Y. Korchin, V. Pascalutsa and D. Van Neck, Phys. Lett. B 384, 13 (1996); A. Y. Korchin, O. Scholten and R. G. E. Timmermans, ibid. 438, 1 (1998); A. Usov and O. Scholten, Phys. Rev. C 72, $025205(2005)$.

[10] T. Feuster and U. Mosel, Phys. Rev. C 59, 460 (1999); G. Penner and U. Mosel, ibid. 66, 055211 (2002); H. Lenske, V. Shklyar and U. Mosel, "Meson Production on the Nucleon in the Giessen K-Matrix Approach," arXiv:nucl-th/0512044.

[11] D. Drechsel, O. Hanstein, S. S. Kamalov and L. Tiator, Nucl. Phys. A 645, 145 (1999) http://www.kph.uni-mainz.de/MAID.

[12] I. G. Aznauryan, Phys. Rev. C 68, 065204 (2003).

[13] S. Nozawa, B. Blankleider and T.-S. H. Lee, Nucl. Phys. A 513, 459 (1990); T.-S. H. Lee and B. C. Pearce, ibid. 530, 532 (1991); T. Sato and T.-S. H. Lee, Phys. Rev. C 54, 2660 (1996); ibid. 63, 055201 (2001).

[14] Y. Surya and F. Gross, Phys. Rev. C 53, 2422 (1996); ibid. 47, 703 (1993).

[15] S. S. Kamalov and S. N. Yang, Phys. Rev. Lett. 83, 4494 (1999); S. S. Kamalov, G. Y. Chen, S. N. Yang, D. Drechsel and L. Tiator, Phys. Lett. B 522, 27 (2001); L. Tiator, D. Drechsel, S. S. Kamalov and S. N. Yang, Eur. Phys. J. A 17, 357 (2003).

[16] M. G. Fuda and H. Alharbi, Phys. Rev. C 68, 064002 (2003).

[17] V. Pascalutsa and J. A. Tjon, Phys. Lett. B 435, 245 (1998); Phys. Rev. C 61, 054003 (2000); ibid. 70, 035209 (2004). 
[18] G. Caia, V. Pascalutsa, J. A. Tjon and L. E. Wright, Phys. Rev. C 70, 032201(R) (2004); G. Caia, L. E. Wright and V. Pascalutsa, ibid. 72, 035203 (2005).

[19] S. Weinberg, Physica A 96, 327 (1979).

[20] J. Gasser and H. Leutwyler, Annals Phys. 158, 142 (1984).

[21] E. Jenkins and A. V. Manohar, Phys. Lett. B 255, 558 (1991); H.-B. Tang and P. Ellis, ibid. 387, 9 (1996); T. Hemmert, B. R. Holstein and J. Kambor, ibid. 395, 89 (1997); N. Fettes and U. G. Meissner, Nucl. Phys. A 679, 629 (2001); C. Hacker, N. Wies, J. Gegelia and S. Scherer, Phys. Rev. C 72, 055203 (2005).

[22] V. Pascalutsa and D. R. Phillips, Phys. Rev. C 67, 055202 (2003).

[23] V. Pascalutsa and M. Vanderhaeghen, Phys. Rev. Lett. 95, 232001 (2005).

[24] V. Pascalutsa and M. Vanderhaeghen, Phys. Rev. Lett. 94, 102003 (2005).

[25] C. Alexandrou et al., Phys. Rev. Lett. 94, 021601 (2005).

[26] V. Pascalutsa and M. Vanderhaeghen, "The nucleon and Delta-resonance masses in relativistic chiral effective-field theory," arXiv:hep-ph/0511261.

[27] J. Gasser, M. E. Sainio and A. Svarc, Nucl. Phys. B 307, 779 (1988).

[28] R. F. Dashen, E. Jenkins and A. V. Manohar, Phys. Rev. D 49, 4713 (1994) [Erratum-ibid. D 51, 2489 (1995)].

[29] M. N. Butler, M. J. Savage and R. P. Springer, Nucl. Phys. B 399, 69 (1993).

[30] V. Pascalutsa, Phys. Rev. D 58, 096002 (1998); V. Pascalutsa and R. G. E. Timmermans, Phys. Rev. C 60, 042201(R) (1999).

[31] V. Pascalutsa, Phys. Lett. B 503, 85 (2001).

[32] V. Pascalutsa, PhD Thesis (University of Utrecht, 1998) [Hadronic J. Suppl. 16, 1 (2001)], Ch. 3.

[33] J. Gegelia and G. Japaridze, Phys. Rev. D 60, 114038 (1999); J. Gegelia, G. Japaridze and X. Q. Wang, J. Phys. G 29, 2303 (2003).

[34] H. F. Jones and M. D. Scadron, Ann. Phys. 81, 1 (1973).

[35] A. M. Bincer, Phys. Rev. 118, 855 (1960); S. D. Drell and H. R. Pagels, ibid. 140, B397 (1965).

[36] B. R. Holstein, V. Pascalutsa and M. Vanderhaeghen, Phys. Rev. D 72, 094014 (2005).

[37] K. M. Watson, Phys. Rev. 95, 228 (1954).

[38] R. A. Arndt, W. J. Briscoe, I. I. Strakovsky, and R. L. Workman, Phys. Rev. C 66, 055213 (2002) [SAID solutions at http://gwdac .phys.gwu .edu.

[39] C. Kunz et al., Phys. Lett. B 564, 21 (2003).

[40] T. Pospischil et al., Phys. Rev. Lett. 86, 2959 (2001).

[41] D. Elsner et al., "Measurement of the LT-asymmetry in pi0 electroproduction at the energy of the $\operatorname{Delta}(1232)$ resonance," arXiv:nucl-ex/0507014.

[42] C. E. Carlson, Phys. Rev. D 34, 2704 (1986).

[43] C. W. Bernard et al., Phys. Rev. D 64, 054506 (2001).

[44] I. C. Cloet, D. B. Leinweber and A. W. Thomas, Phys. Lett. B 563, 157 (2003); R. D. Young, D. B. Leinweber and A. W. Thomas, Nucl. Phys. Proc. Suppl. 129, 290 (2004).

[45] C. Alexandrou et al., "A study of the N to Delta transition form factors in full QCD," arXiv:hep-lat/0509140.

[46] C. W. Kao, "The pion electroproduction in the Delta(1232) region," arXiv:nucl-th/9910069. 


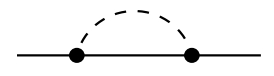

FIG. 1: Nucleon self-energy to one loop.

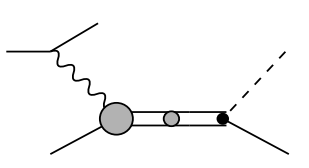

(a)

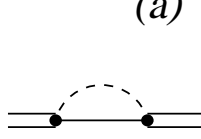

(c)
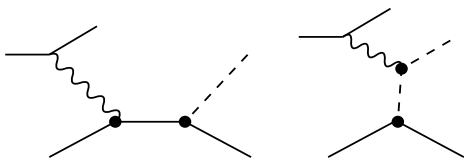

(b)

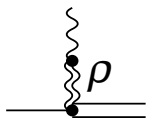

(d)

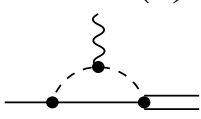

(e)
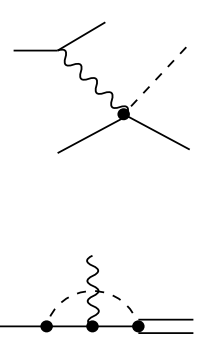

(f)

FIG. 2: Diagrams for the $e N \rightarrow e \pi N$ reaction at NLO in the $\delta$-expansion, considered in this work. Double lines represent the $\Delta$ propagators.

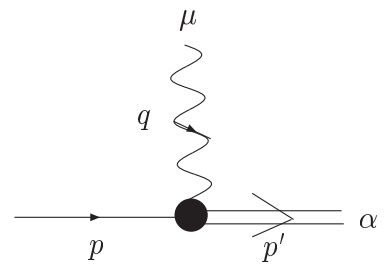

FIG. 3: $\gamma^{*} N \Delta$ vertex. The four-momenta of the nucleon $(\Delta)$ and of the photon are given by $p\left(p^{\prime}\right)$ and $q \equiv p^{\prime}-p$ respectively. The four-vector index of the spin $3 / 2$ field is given by $\alpha$, and $\mu$ is the four-vector index of the photon field. 


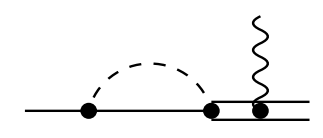

FIG. 4: The higher-order $\gamma N \Delta$ vertex correction required by the electromagnetic current conservation.

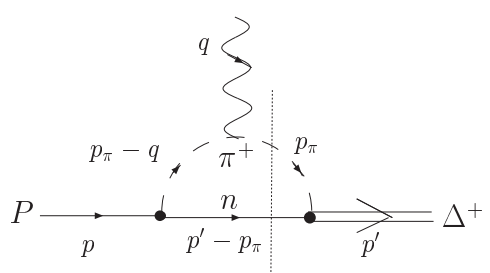

(a)

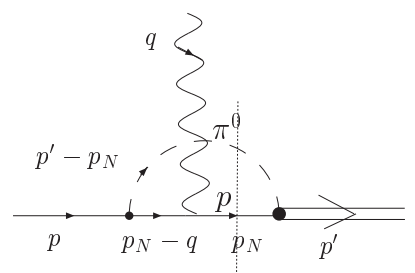

(b)

FIG. 5: Absorptive part of the $\gamma p \Delta^{+}$vertex. Diagram (a) : $\pi^{+} n$ loop where the photon couples to the $\pi^{+}$; diagram (b) : $\pi^{0} p$ loop where the photon couples to the charge of the proton. The vertical dotted lines indicate that the $\pi N$ intermediate state is taken on-shell.

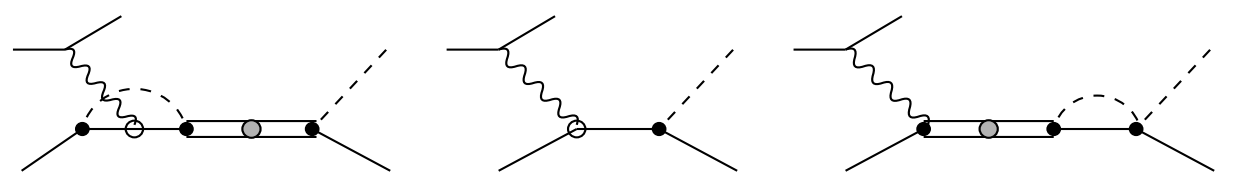

FIG. 6: Examples of $\mathrm{N}^{2} \mathrm{LO}$ contributions to the $e N \rightarrow e \pi N$ reaction neglected in this work. Open circles denote the e.m. coupling to the anomalous magnetic moment of the nucleon. 

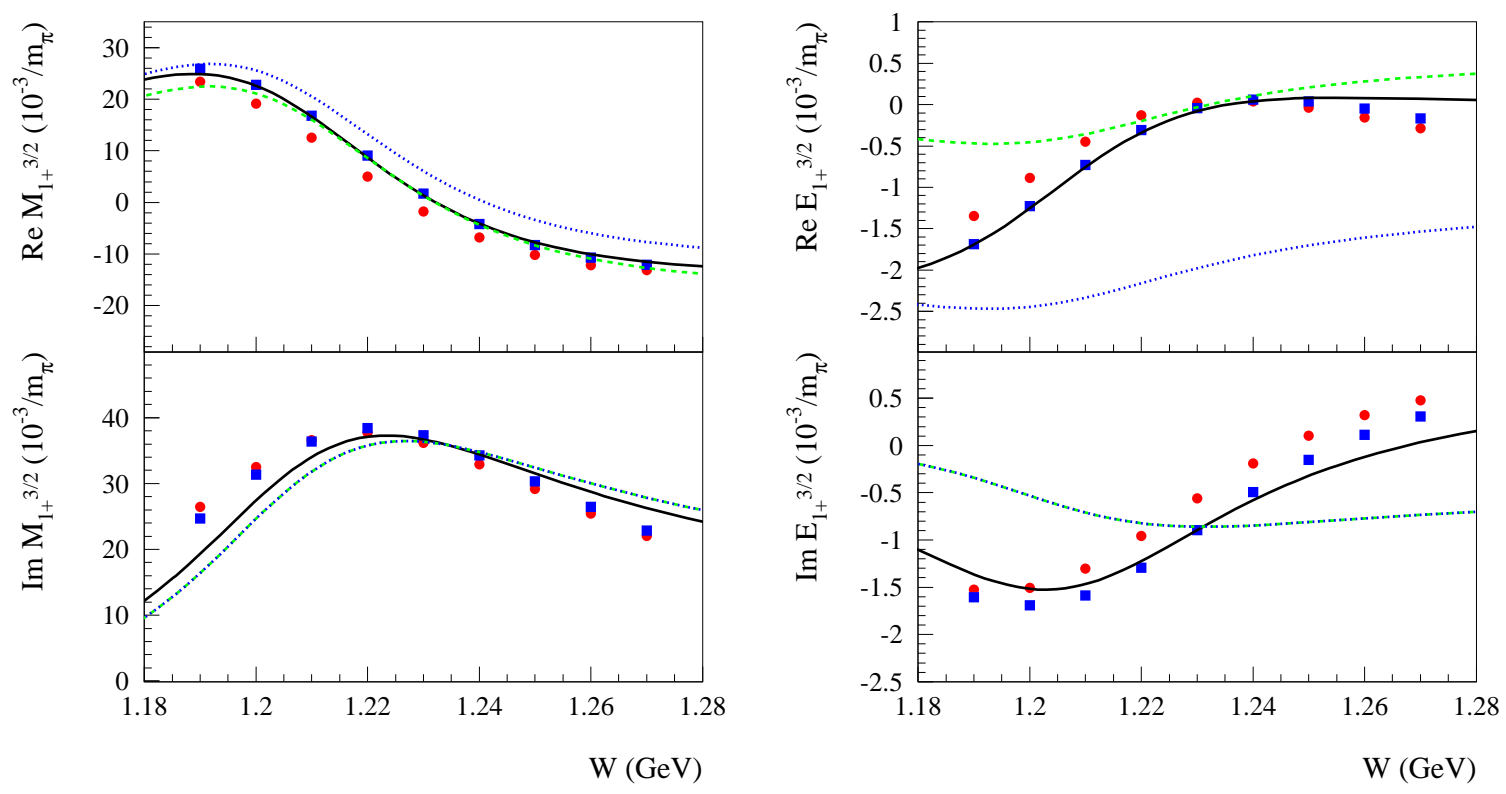

FIG. 7: (Color online) Multipole amplitudes $M_{1+}^{(3 / 2)}$ (left panel) and $E_{1+}^{(3 / 2)}$ (right panel) for pion photoproduction as function of the invariant mass $W$ of the $\pi N$ system. Green dashed curves: $\Delta$ contribution without the $\gamma N \Delta$-vertex corrections, [i.e., Fig. [2(a) without Fig. [2(e, f)]. Blue dotted curves: adding the Born contributions, Fig. 2(b), to the dashed curves. Black solid curves: complete NLO calculation, includes all graphs from Fig. 2, In all curves the low-energy parameters are chosen as : $g_{M}=2.9, g_{E}=-1.0$. The data point are from the SAID analysis (FA04K) 38] (red circles), and from the MAID 2003 analysis [11] (blue squares). 

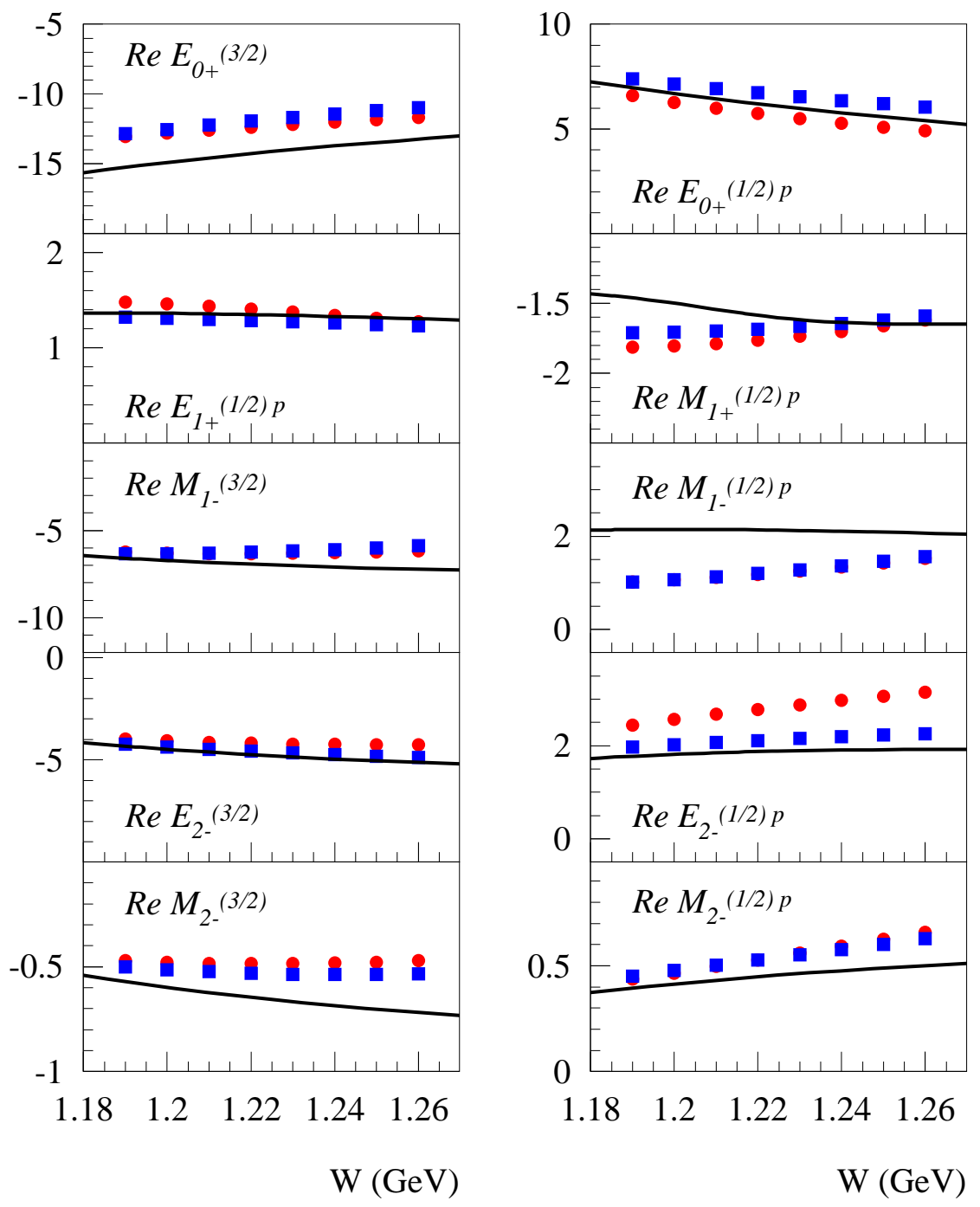

FIG. 8: (Color online) Non-resonant multipole amplitudes (in units $10^{-3} / m_{\pi}$ ) for pion photoproduction as function of the invariant mass $W$ of the $\pi N$ system. The solid curves result from our NLO calculation. The data points are from the SAID analysis (FA04K) 38] (red circles), and from the MAID 2003 analysis [1]] (blue squares). 

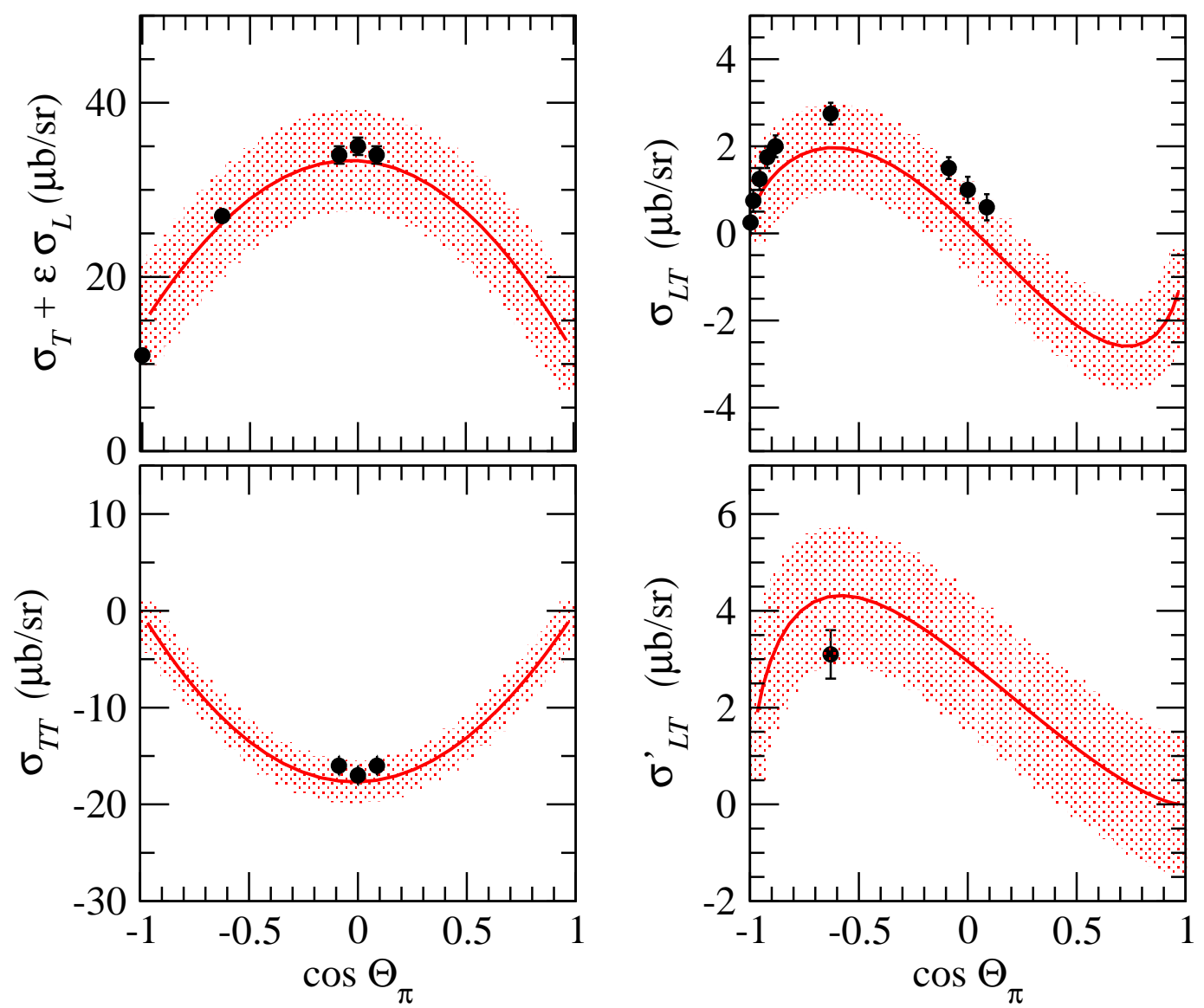

FIG. 9: (Color online) The NLO results for the $\Theta_{\pi}$ dependence of the $\gamma^{*} p \rightarrow \pi^{0} p$ cross sections at $W=1.232 \mathrm{GeV}$ and $Q^{2}=0.127 \mathrm{GeV}^{2}$. The theoretical error bands are described in the text. Data points are from BATES experiments [3, 39]. 

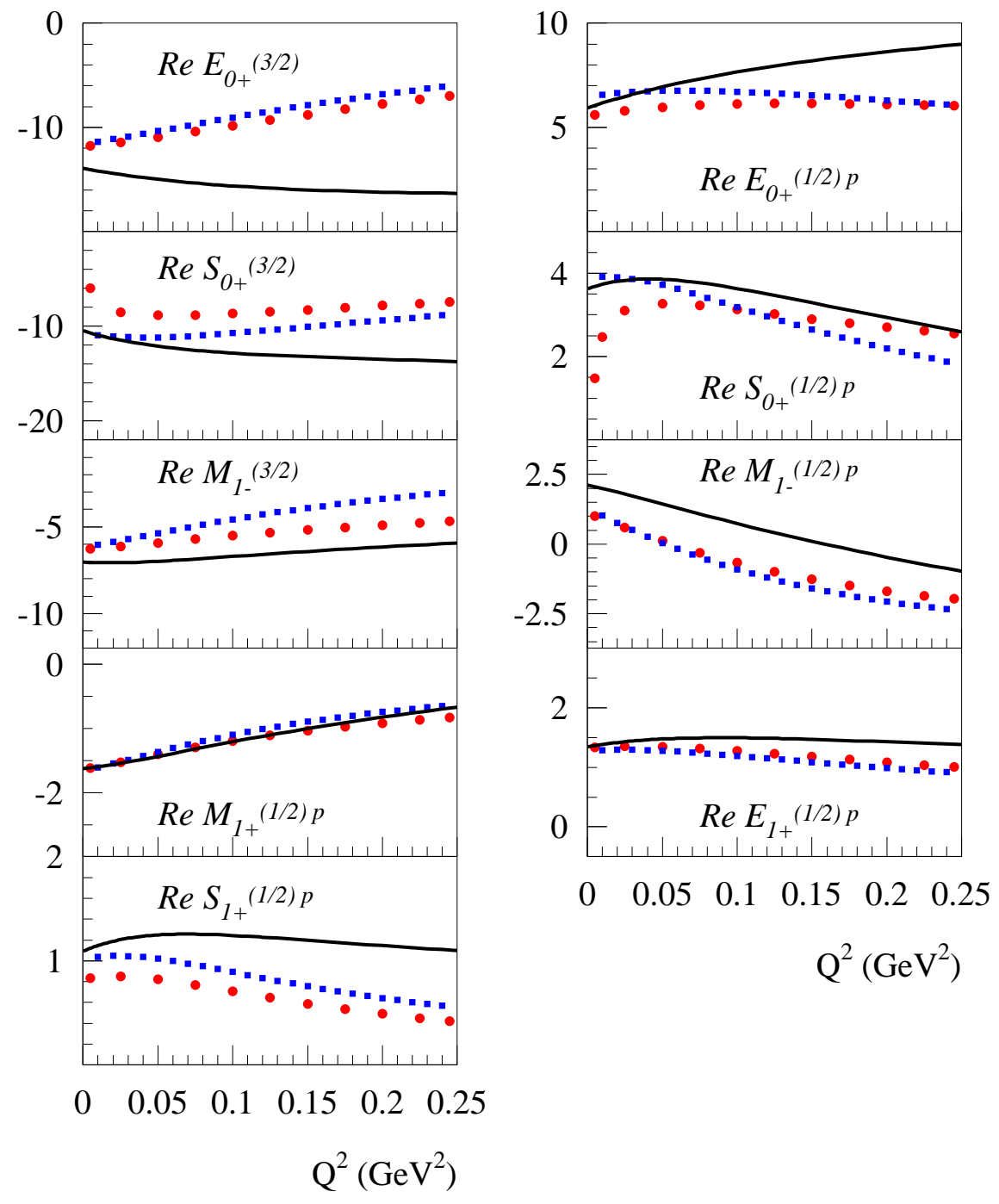

FIG. 10: (Color online) Non-resonant multipole amplitudes (in units $10^{-3} / m_{\pi}$ ) for pion electroproduction at the resonance position $(W=1.232 \mathrm{GeV})$ as function of $Q^{2}$. The curves are the results of our NLO calculation. The data points are from the SAID analysis (FA04K) 38] (red circles), and from the MAID 2003 analysis 11] (blue squares). 


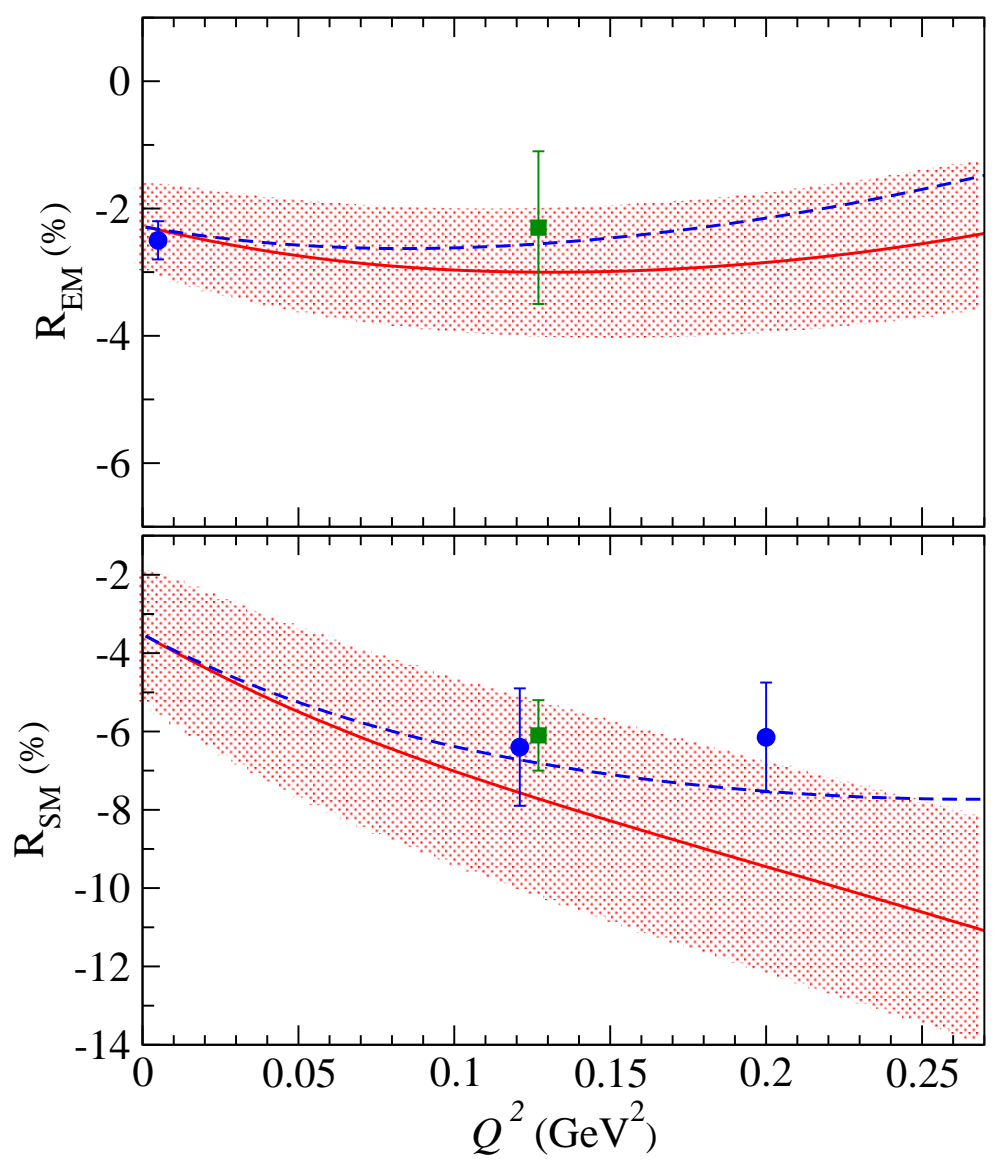

FIG. 11: (Color online) $Q^{2}$ dependence of the NLO results (solid curves) for $R_{E M}$ (upper panel) and $R_{S M}$ (lower panel). The blue dashed curves represent a phenomenological estimate of $\mathrm{N}^{2} \mathrm{LO}$ effects by including $Q^{2}$-dependence in $g_{E}$ according to Eq. (60). The blue circles are data points from MAMI for $R_{E M}[1]$, and $R_{S M}$ [40, 41]. The green squares are data points from BATES [3]. 


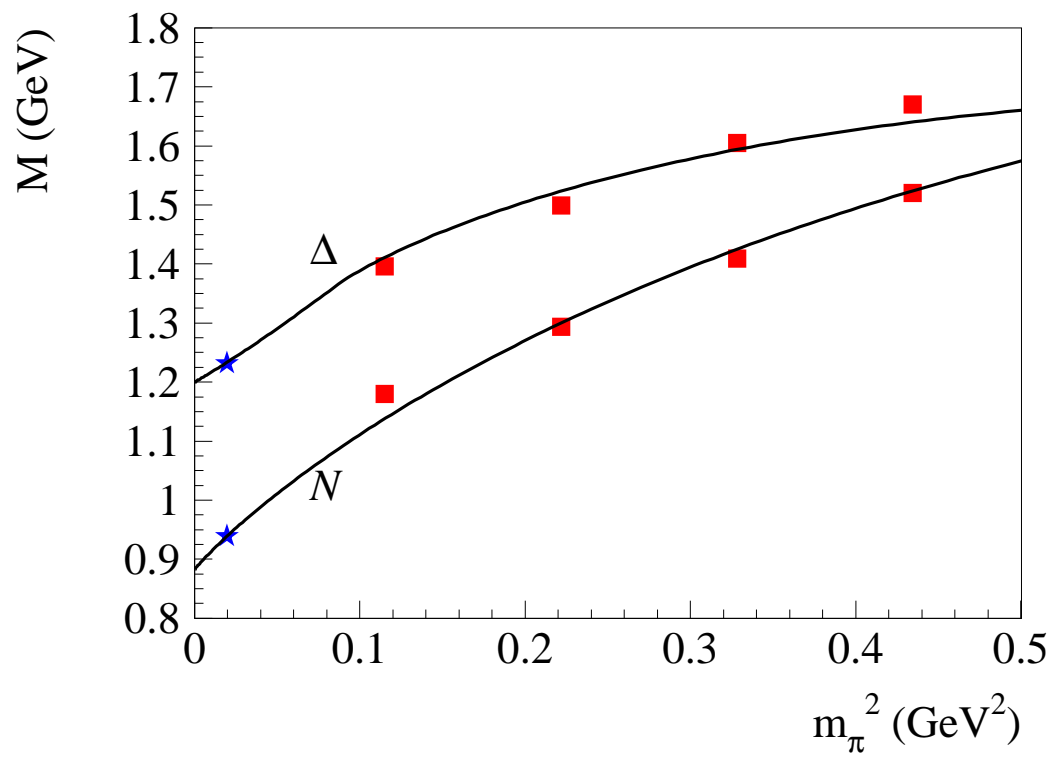

FIG. 12: (Color online) Pion-mass dependence of the nucleon and $\Delta(1232)$ masses. The curves are two-parameter expressions for the $\pi N$ loop contributions to $M_{N}$ and $M_{\Delta}$ according to Eqs. (19) and (25) respectively, using $M_{N}^{(0)}=0.883 \mathrm{GeV}, c_{1 N}=-0.87 \mathrm{GeV}^{-1}$, and $M_{\Delta}^{(0)}=1.20 \mathrm{GeV}, c_{1 \Delta}=$ $-0.40 \mathrm{GeV}^{-1}$ respectively. The red squares are lattice results from the MILC Collaboration [43]. The stars represent the physical mass values. 


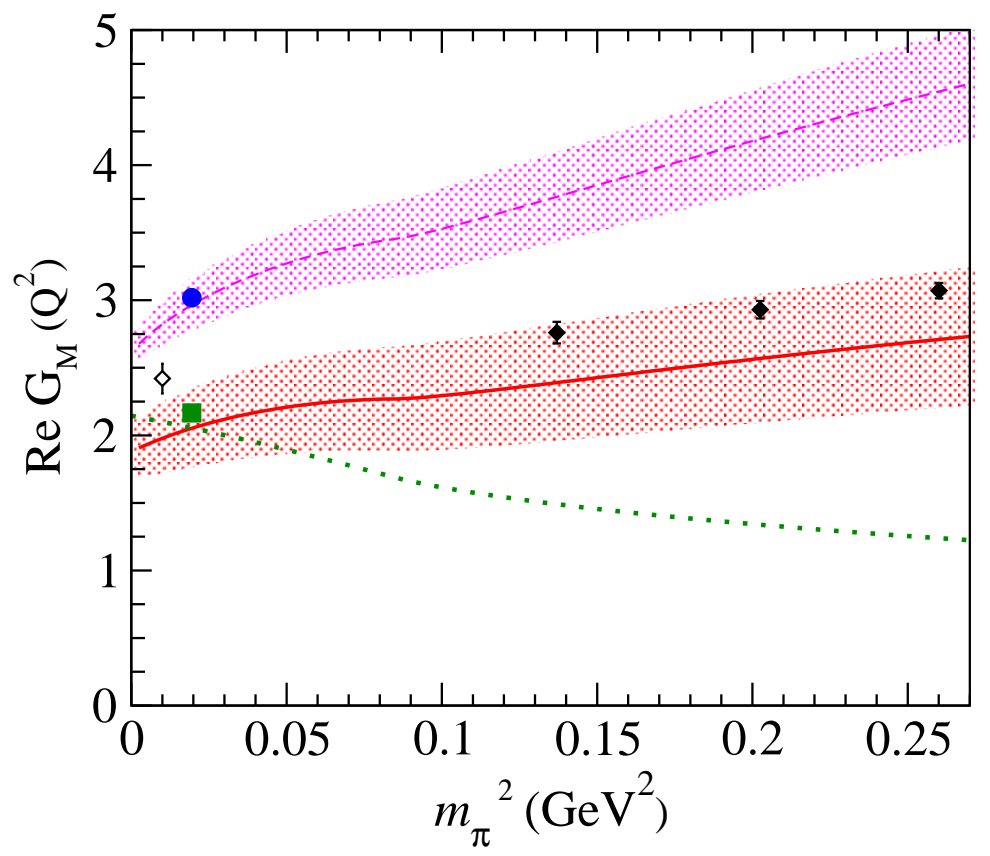

FIG. 13: (Color online) The $m_{\pi}$-dependence of the real part of the Jones-Scadron $\gamma N \Delta$ form factor $G_{M}^{*}$ for $Q^{2}=0$ and $Q^{2}=0.127 \mathrm{GeV}^{2}$. The solid (dashed) curves are the NLO results for $Q^{2}=0.127 \mathrm{GeV}^{2}\left(Q^{2}=0\right)$ respectively, including the $m_{\pi}$ dependence of $M_{N}$ and $M_{\Delta}$. The green dotted curve is the corresponding result for $Q^{2}=0.127 \mathrm{GeV}^{2}$ where the $m_{\pi}$ dependence of $M_{N}$ and $M_{\Delta}$ is not included. The blue circle for $Q^{2}=0$ is a data point from MAMI 1], and the green square for $Q^{2}=0.127 \mathrm{GeV}^{2}$ is a data point from BATES [3]. The three filled black diamonds at larger $m_{\pi}$ are lattice calculations 25] for $Q^{2}$ values of $0.125,0.137$, and $0.144 \mathrm{GeV}^{2}$ respectively, whereas the open diamond near $m_{\pi} \simeq 0$ represents their extrapolation assuming linear dependence in $m_{\pi}^{2}$. 


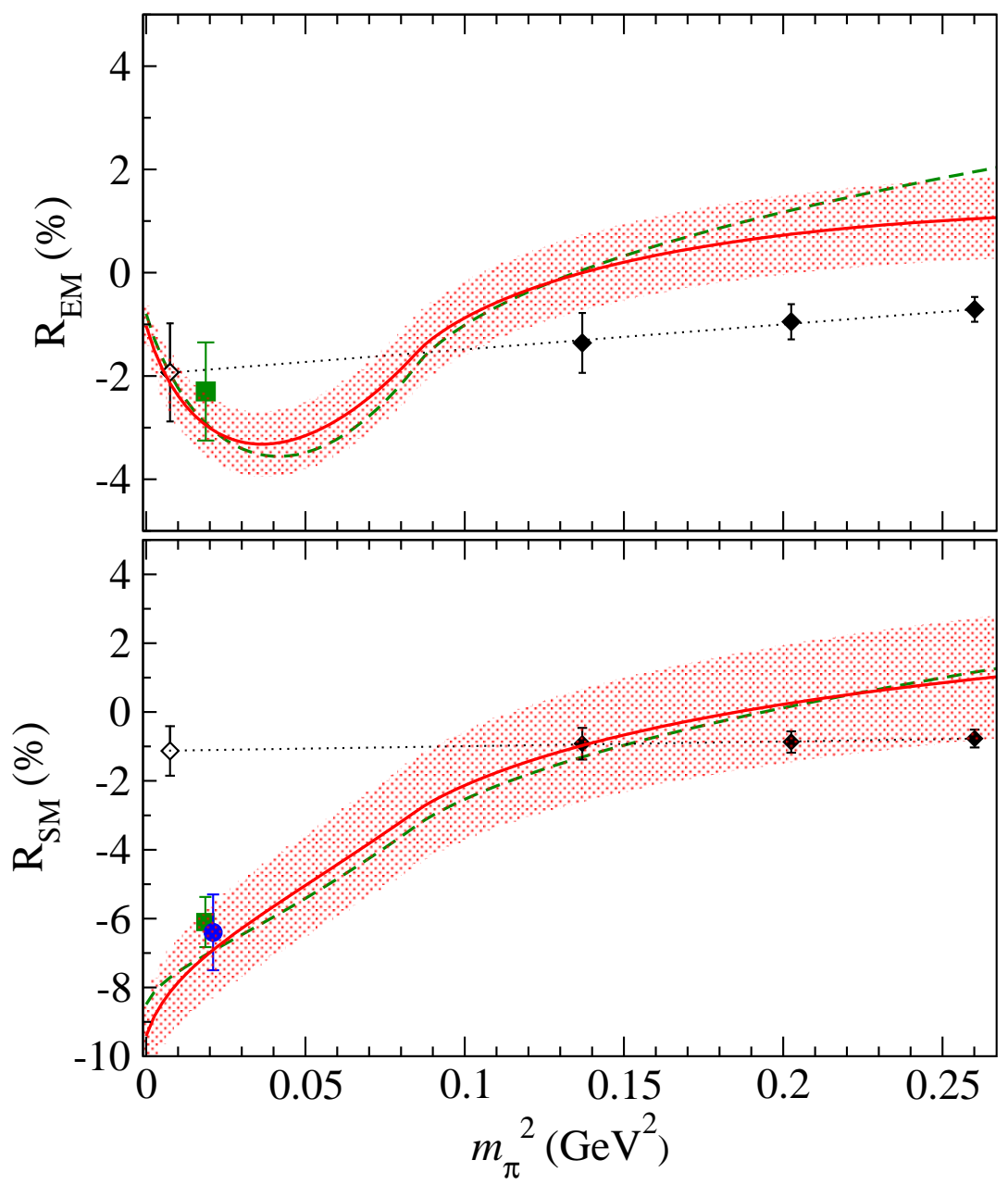

FIG. 14: (Color online) $m_{\pi}$ dependence of the NLO results at $Q^{2}=0.1 \mathrm{GeV}^{2}$ for $R_{E M}$ (upper panel) and $R_{S M}$ (lower panel). The blue circle is a data point from MAMI [40], the green squares are data points from BATES [3]. The three filled black diamonds at larger $m_{\pi}$ are lattice calculations [25], whereas the open diamond near $m_{\pi} \simeq 0$ represents their extrapolation assuming linear dependence in $m_{\pi}^{2}$. Red solid curves: NLO result when accounting for the $m_{\pi}$ dependence in $M_{N}$ and $M_{\Delta}$; green dashed curves: NLO result of Ref. [23], where the $m_{\pi}$-dependence of $M_{N}$ and $M_{\Delta}$ was not accounted for. 